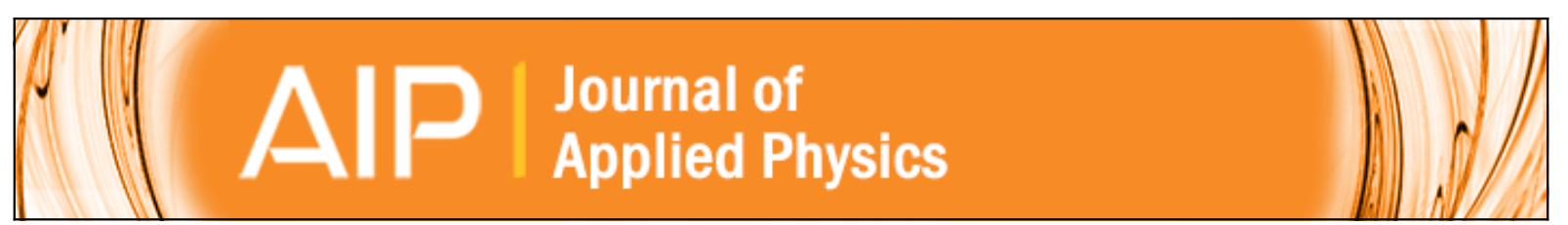

Asymptotic and numerical prediction of current-voltage curves for an organic bilayer solar cell under varying illumination and comparison to the Shockley equivalent circuit J. M. Foster, J. Kirkpatrick, and G. Richardson

Citation: Journal of Applied Physics 114, 104501 (2013); doi: 10.1063/1.4820567

View online: http://dx.doi.org/10.1063/1.4820567

View Table of Contents: http://scitation.aip.org/content/aip/journal/jap/114/10?ver=pdfcov

Published by the AIP Publishing

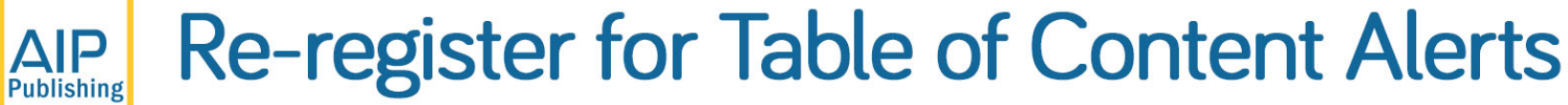




\title{
Asymptotic and numerical prediction of current-voltage curves for an organic bilayer solar cell under varying illumination and comparison to the Shockley equivalent circuit
}

\author{
J. M. Foster, ${ }^{1, a)}$ J. Kirkpatrick, ${ }^{2}$ and G. Richardson ${ }^{1, b)}$ \\ ${ }^{1}$ School of Mathematics, University of Southampton, Southampton, Hampshire SO17 1BJ, United Kingdom \\ ${ }^{2}$ Department of Physics, University of Oxford, Oxford, Oxfordshire OXI 3PU, United Kingdom
}

(Received 12 March 2013; accepted 22 August 2013; published online 9 September 2013)

\begin{abstract}
In this study, a drift-diffusion model is used to derive the current-voltage curves of an organic bilayer solar cell consisting of slabs of electron acceptor and electron donor materials sandwiched together between current collectors. A simplified version of the standard drift-diffusion equations is employed in which minority carrier densities are neglected. This is justified by the large disparities in electron affinity and ionisation potential between the two materials. The resulting equations are solved (via both asymptotic and numerical techniques) in conjunction with (i) Ohmic boundary conditions on the contacts and (ii) an internal boundary condition, imposed on the interface between the two materials, that accounts for charge pair generation (resulting from the dissociation of excitons) and charge pair recombination. Current-voltage curves are calculated from the solution to this model as a function of the strength of the solar charge generation. In the physically relevant power generating regime, it is shown that these current-voltage curves are well-approximated by a Shockley equivalent circuit model. Furthermore, since our drift-diffusion model is predictive, it can be used to directly calculate equivalent circuit parameters from the material parameters of the device. (C) 2013 AIP Publishing LLC. [http://dx.doi.org/10.1063/1.4820567]
\end{abstract}

\section{INTRODUCTION}

Organic photovoltaic devices are a promising new technology ${ }^{14}$ which provide the prospect of very cheap solar cells that can be produced on a large scale. ${ }^{15}$ These organic devices present several advantages over their traditional inorganic counterparts: (i) ease of manufacture using high throughput techniques such as roll to roll printing, (ii) low cost materials, and (iii) mechanical flexibility. Currently, rapid progress is being made by a number of companies towards the goal of making cells with energy efficiencies sufficient to compete with standard inorganic devices in the commercial market. ${ }^{34}$

Solar cells operate by absorbing light to create excited charge pairs (known as excitons), then separating these charges and transporting them to current collectors where they may be used to deliver electrical power to an external circuit. Charge separation is typically driven by the diode like properties of the device. These facilitate the easy flow of the current in one direction, but not the other, and thus lead to the preferential deposition of positive charge on one current collector and negative charge on the other. In inorganic solar cells, the diode like behaviour of the device is achieved by doping the materials asymmetrically. In contrast, in organic devices, this behaviour is achieved by choosing two organic semiconductors (the donor and acceptor) with markedly different preferences for holes and electrons so that holes preferentially separate into the donor (small ionization potential) while conduction electrons preferentially separate into the acceptor (high electron affinity). Charge generation within inorganic materials occurs when a

\footnotetext{
${ }^{a}$ Electronic mail: j.m.foster@soton.ac.uk

${ }^{b}$ Currently at: Tessella Ltd., 26 The Quadrant, Abingdon Science Park, Abingdon, Oxfordshire OX14 3YS, United Kingdom
}

sufficiently energetic photon is absorbed to create a weakly bound exciton that can readily separate into a free conduction electron and a free hole. In contrast, in an organic solar cell, photon absorption typically results in the creation of a strongly bound exciton. This can diffuse and may either recombine, releasing its energy (as heat or light), or reach the acceptordonor interface where the differences in ionization potential and electron affinity between the two materials may be sufficient to separate it into a free hole in the donor and a free electron in the acceptor. Once separated these free charges may either recombine on the acceptor-donor interface (losing their energy) or be transported to the current collectors where their energy can be harvested.

A major problem with organic solar cells is that the exciton diffusion length (before recombination) is typically very small, around $10 \mathrm{~nm},{ }^{14}$ while the thickness of organic semiconductor required to absorb a significant portion of the incident sunlight is much larger, typically around $200 \mathrm{~nm} .^{14}$ Thus, a simple bilayer organic device (see Figure 1) cannot both absorb a significant portion of the incident light and ensure that a significant portion of the excitons generated reach the acceptor-donor interface where their charges can separate. Hence, the efficiency of bilayer devices is poor. ${ }^{7,29,36}$ In order to circumvent this difficulty, it is customary to construct bulk heterojunctions which are sufficiently thick $(\sim 30-300 \mathrm{~nm})$ to absorb a significant portion of the incident sunlight but also possess a highly convoluted acceptor-donor interface with oscillations on the exciton diffusion lengthscale $(\sim 10 \mathrm{~nm})$; this ensures that most of the excitons generated reach the interface before recombining. Some images, obtained using scanning electron microscopy, of real bulk heterojunctions with a fully 3-dimensional structure are shown in Pautmeier et al. ${ }^{28}$ Thus, 

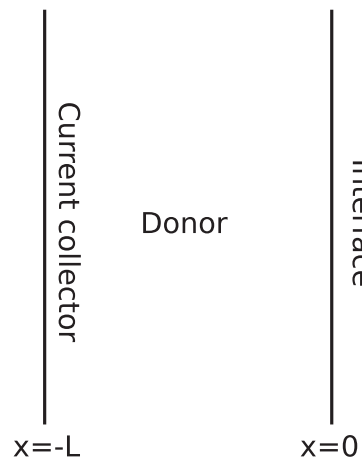

$x=-L$
Acceptor

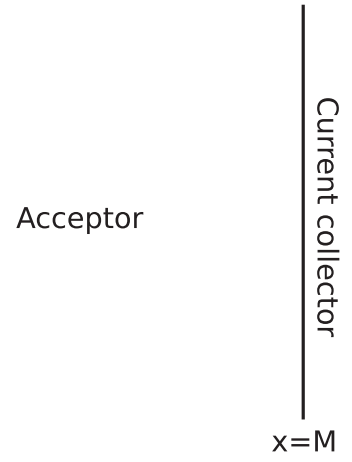

$x=M$

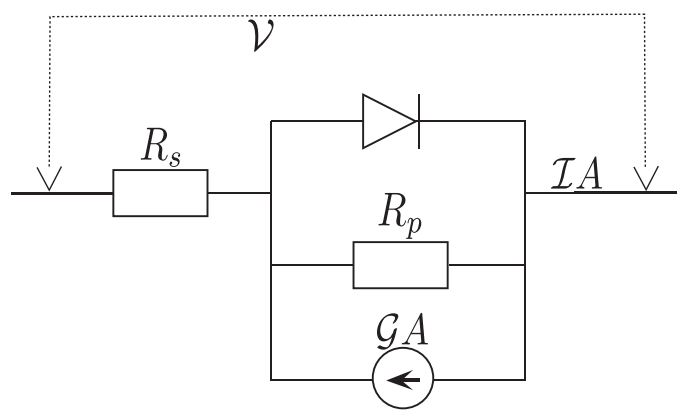

FIG. 2. A schematic of the Shockley equivalent circuit.

FIG. 1. A schematic of a bilayer organic solar cell.

much of the current theoretical research into organic solar cells has concentrated on the role of morphology in bulk heterojunctions and, due to the inherently complex geometry, has required a heavily numerical approach. ${ }^{1,3,24}$ An alternative approach, which has been commonly adopted, is to describe highly heterogeneous acceptor-donor microstructure as an effective medium. ${ }^{10,12,20}$ This has the advantage that the interface does not appear explicitly in the problem so that the device is described by a set of equations with one spatial dimension but has the disadvantage that the link between interface morphology and device behaviour is lost. A possible way of reconciling these two approaches, which will be explored in a future work, is to use asymptotic homogenisation techniques, ${ }^{5,31}$ to systematically derive effective medium equations for the heterostructure from a detailed microscale model. ${ }^{1,3,24}$

Here, we extend previous work on the behaviour of a simple bilayer device in the dark ${ }^{32}$ to investigate the effects of significant levels of solar generation on its current-voltage curve. This enables us to test the validity of our model unencumbered by the additional complexities associated with the complex morphology of a bulk heterojunction device. The central approach that we use is the method of matched asymptotics. This allows us to derive analytic approximations for the current-voltage curves of bilayer devices in all physically relevant power generating regimes. Furthermore, we show that these expressions are asymptotic to the widely used Shockley equivalent circuit (SEC) model and that they compare favourably to a fully numerical solution of the drift-diffusion model. The SEC describes the behaviour of a solar cell by an equivalent circuit consisting of a diode in series with a resistor and in parallel with a shunt resistor and a current source (see Figure 2). It is most usually written in the form

$$
\mathcal{I}=\frac{R_{p}}{R_{p}+R_{s}} \times\left(\mathcal{I}_{0}\left[\exp \left(\frac{\mathcal{V}-\mathcal{I} R_{s} A}{\mathcal{N} k T / q}\right)-1\right]-\mathcal{G}-\frac{\mathcal{V}}{R_{p} A}\right) .
$$

Here, $\mathcal{I}$ is the current density in the device, $\mathcal{V}$ is the applied voltage, $R_{p}$ and $R_{s}$ are the shunt and series resistivities, respectively, $\mathcal{I}_{0}$ is the reverse saturation current density of the diode, $\mathcal{G}$ is the photo-current density, $A$ is the area of the cell, $\mathcal{N}$ is the ideality factor of the diode, $k T$ is the thermal energy, and $-q$ is the charge on an electron. The most notable difference between the predictions of the drift-diffusion

model proposed here and the SEC is that our model does not predict a shunt resistance (i.e., $R_{p} \rightarrow \infty$ in Eq. (1)). This shunt resistance could be an artefact of either (i) pinhole defects, i.e., when a cell has been manufactured in such a way that a region of donor (or alternatively acceptor) spans the region between both current collectors and hence creates a short circuit ${ }^{11,22}$ or (ii) the presence of minority carriers (in either acceptor or donor) acting to short circuit the device. ${ }^{23,38}$ However, given the large differences in the ionization potentials and electron affinities between the donor and acceptor materials used in real bilayer devices, we estimate that shorting currents carried by minority carriers are insignificant in practice.

\section{PROBLEM FORMULATION}

The main physical processes taking place within an organic solar cell are charge transport (within donor and acceptor materials) and the generation and recombination of charges at the acceptor-donor interface. . $^{3,8,12}$

Various models have been applied to the transport process, such as multiple trap models, ${ }^{16}$ atomistic models, ${ }^{21}$ and Gaussian disorder models. ${ }^{30}$ Here, we elect to use the driftdiffusion equations $\mathrm{s}^{35}$ in order to provide a macroscopic description of the hopping process whereby free charges (electrons or holes) move from one molecule to the next. As previously noted by Richardson et al., the large differences in ionization potential and electron affinity between the acceptor and donor justify the assumptions of zero free electron density in the donor and zero hole density in the acceptor. ${ }^{32}$

The main mechanisms allowing charge to pass from the acceptor to the donor are (non-geminate) recombination of a hole from the donor with a free electron from the acceptor and generation of charge pairs at the interface. Generation results both from (i) solar generation in which an exciton pair (generated from absorbed light energy) dissociates on the interface into a hole in the donor and an electron in the acceptor and (ii) thermal emission of electron hole pairs from the interface. The net effect of the combination of these two mechanisms is to transport charge between acceptor and donor. Here, we initially model interface recombination between charge carriers using a Shockley-Read-Hall (SRH) condition noting that this leads to an ideality factor $\mathcal{N}=2$ in contrast to Langevin recombination $(R \propto n p)$ which models the direct recombination of charges and gives $\mathcal{N}=1$. Experimental measurements of the ideality factor ${ }^{18}$ typically lie between 1 and 2, for example, Credgington et al. 
measured an ideality factor of $\sim 1.69$ in a polymer/ $\mathrm{C}_{60}$ bilayer, ${ }^{6}$ indicating that in reality, both Langevin and $\mathrm{SRH}$ recombination may be taking place. ${ }^{17}$ In view of this variability, we consider a generalised recombination condition in Sec. V, that is capable of describing a range of ideality factors, and assess how this alters the predicted behaviour of the device.

\section{A. Bulk equations}

Charge carrier motion in both the acceptor and donor materials is modelled by drift-diffusion equations. We exploit the strong preference of electrons for the acceptor and holes for donor to make the simplification that the electron density in the donor and the hole density in the acceptor are both vanishingly small. ${ }^{32}$ It follows that electron-hole recombination occurs only at the acceptor-donor interface and that the resulting conservation equations for the hole density $p$ (in the donor $-L<x<0$ ) and the electron density $n$ (in the acceptor $0<x<M$ ) are

$$
\begin{gathered}
q \frac{\partial p}{\partial t}+\frac{\partial J}{\partial x}=0 \text { for } \quad-L<x<0, \\
q \frac{\partial n}{\partial t}-\frac{\partial J}{\partial x}=0 \text { for } \quad 0<x<M .
\end{gathered}
$$

Here, $J$ is the current density and is related to the flux of holes in the donor $F_{p}$ by $J=q F_{p}$ and to the flux of electrons in the acceptor $F_{n}$ by $J=-q F_{n}$ so that

$$
\begin{gathered}
J=-q D_{p}\left(\frac{\partial p}{\partial x}+\frac{q}{k T} p \frac{\partial \phi}{\partial x}\right) \text { for } \quad-L<x<0 \\
J=q D_{n}\left(\frac{\partial n}{\partial x}+\frac{q}{k T} n \frac{\partial \phi}{\partial x}\right) \text { for } \quad 0<x<M
\end{gathered}
$$

where $\phi, k$, and $T$ are electric potential, Boltzmann's constant, and the absolute temperature, respectively. The diffusion coefficients of electrons and holes are $D_{n}$ and $D_{p}$, respectively-these are related to the electron and hole mobilities, $\mu_{n}$ and $\mu_{p}$, via the Einstein relation, $D_{n, p}=k T \mu_{n, p} / q$. The Einstein relation is applicable when electrons and holes move independently, i.e., do not form bound pairs. The electric potential obeys Poisson's equation

$$
\frac{\partial}{\partial x}\left(\epsilon \frac{\partial \phi}{\partial x}\right)=\left\{\begin{array}{ccc}
-q p & \text { for } & -L<x<0 \\
q n & \text { in } & 0<x<M
\end{array},\right.
$$

where

$$
\epsilon=\left\{\begin{array}{ccc}
\epsilon_{d} & \text { for } & -L<x<0 \\
\epsilon_{a} & \text { in } & 0<x<M
\end{array},\right.
$$

where $\epsilon$ is the absolute permittivity.

\section{B. Jump and boundary conditions}

At the acceptor-donor interface, continuity of electric potential and the normal component of electric displacement are imposed in the standard fashion

$$
\left.\phi\right|_{x=0^{-}}=\left.\phi\right|_{x=0^{+}},\left.\quad \epsilon_{d} \frac{\partial \phi}{\partial x}\right|_{x=0^{-}}=\left.\epsilon_{a} \frac{\partial \phi}{\partial x}\right|_{x=0^{+}} .
$$

The effect of recombination of electron-hole pairs at the acceptor-donor interface is initially modelled by a SRH term while the contribution to the current density due to exciton dissociation at the interface is denoted by $-G$ so that

$$
\begin{aligned}
\left.J\right|_{x=0^{-}} & =\left.J\right|_{x=0^{+}} \\
& =K_{0}\left(\frac{\left.\left.n\right|_{x=0^{+}} p\right|_{x=0^{-}}-N_{D}^{2} \exp \left(-E_{g} / k T\right)}{1+\left.u_{1} n\right|_{x=0^{+}}+\left.u_{2} p\right|_{x=0^{-}}}\right)-G .
\end{aligned}
$$

The exact details of exciton generation, transport, and dissociation (on the interface) affect only the magnitude of $G$ and will not be considered further other than to remark that exciton dissociation is typically thought to occur in a two step process in which excitons incident on the interface first dissociate into interface-bound geminate pairs before dissociating, under the action of thermal excitation, into free charges. ${ }^{13,27,40}$ In the case of polymer/ $\mathrm{C}_{60}$ cells, the dissociation of geminate pairs is believed to be extremely efficient (with hardly any loss due to recombination). ${ }^{40}$ The constants $K_{0}, N_{D}, u_{1}$, and $u_{2}$ are material parameters, while $E_{g}$ denotes the pseudo-band gap, i.e., the difference in energies between the HOMO of the donor and LUMO of the acceptor.

Finally, the problem is closed by imposing Ohmic boundary conditions on the current collector contacts. On the left contact at $x=-L$, we impose

$$
p=\frac{N_{D}^{2}}{\tilde{n}_{-}} \exp \left(-\frac{E_{g}}{2 k T}+\frac{q V_{b i}}{2 k T}\right), \quad \phi=\frac{V-V_{b i}}{2},
$$

while on the right contact at $x=M$, we impose

$$
n=\tilde{n}_{-} \exp \left(-\frac{E_{g}}{2 k T}+\frac{q V_{b i}}{2 k T}\right), \quad \phi=-\frac{V-V_{b i}}{2},
$$

where $\tilde{n}_{-}, V$, and $V_{b i}$ are a constant scaling factor, the applied voltage across the cell, and the built-in voltage (resulting from the differences in work functions of the current collectors), respectively. The conditions (10) and (11) are the most general form of Ohmic boundary conditions that, together with Eq. (9), give an equilibrium for which $J=G=V=0 .{ }^{32}$ Previous studies ${ }^{33}$ have investigated the effects of tunneling at the contacts. Here, we are primarily interested in scenarios in which the device behaviour is limited by the properties of the semiconductors (and their mutual interface) and not by the contacts and so do not consider tunneling at the contacts further.

\section{Non-dimensionalisation}

Here, we apply a different non-dimensionalisation to that used in Richardson et al., ${ }^{32}$ whose focus was on the dark current of the device, and choose to scale the current density $J$ with a typical photo-generated current density $G_{0}$ (this could, for example, be estimated from the reverse saturation current density of the device). We non-dimensionalise electron- and hole-densities ( $n$ and $p$, respectively) on the electron- and 
hole-fluxes required to carry a current density of magnitude $G_{0}$ and, motivated by our wish to investigate the distinguished limit in which the Debye length is comparable to the device width, choose $G_{0}$ accordingly. This turns out to fix the value of $G_{0}$ close to the current density that would be generated by one Sun. The non-dimensionalisation is as follows (dimensionless quantities are denoted by an over-bar):

$$
\begin{gathered}
J=G_{0} \bar{J}, \quad n=\Pi_{0} \bar{n}, \quad p=\Pi_{0} \bar{p}, \\
\phi=\frac{k T}{q} \bar{\phi}, \quad x=L \bar{x}, \quad t=\frac{q \Pi_{0} L}{G_{0}} \bar{t},
\end{gathered}
$$

where $\Pi_{0}$ and $G_{0}$ are chosen in line with the arguments above such that

$$
\Pi_{0}=\frac{\sqrt{\epsilon_{d} \epsilon_{a}} k T}{q^{2} L^{2}} \text { and } G_{0}=\frac{\sqrt{\epsilon_{d} \epsilon_{a}} \sqrt{D_{d} D_{a}} k T}{q L^{3}} .
$$

By scaling in this manner, we find that the system is characterised by the following dimensionless parameters:

$$
\begin{array}{cc}
\delta=\frac{\Pi_{0} K_{0}}{\left(u_{1}+u_{2}\right) G_{0}}, & \imath=\frac{N_{D} \exp \left(-E_{g} / 2 k T\right)}{\Pi_{0}}, \\
\hat{G}=\frac{G}{G_{0}}, & \hat{\varepsilon}=\sqrt{\frac{\epsilon_{d}}{\epsilon_{a}}}, \\
\kappa=\sqrt{\frac{D_{p}}{D_{n}}}, & N_{-}=\frac{\tilde{n}_{-}}{N_{D}}, \\
\theta=\frac{1}{\Pi_{0}\left(u_{1}+u_{2}\right)}, & \Phi=\frac{u_{1}}{u_{1}+u_{2}}, \\
m=\frac{M}{L}, & \Phi=\frac{q V}{k T}, \\
\Phi_{b i}=\frac{q V_{b i}}{k T} . &
\end{array}
$$

Here, $m$ is the ratio of the widths of the slabs of acceptor and donor, $\hat{\varepsilon}$ is the square root of the ratios of the permittivities of the acceptor and donor, $\kappa$ is the square root of the ratio of the diffusivities in the acceptor and donor, $\theta$ and $U$ are dimensionless parameters characterising the SRH recombination condition, $l$ is the ratio of the intrinsic (thermally generated) carrier concentration to that generated by exciton dissociation, and $\delta$ is the ratio of the current arising from recombination of electrons and holes on the acceptor donor interface to that generated there by the dissociation of excitons into electron-hole pairs.

We remark that the parameters $\delta, \theta, m, U, \Phi$, and $\Phi_{b i}$, as they appear here, are defined identically to those in Richardson et al., ${ }^{32}$ and provided that we take the typical permittivity $\bar{\varepsilon}$ and typical diffusivity $\bar{D}$ (as defined in Richardson et $a l .{ }^{32}$ ) to be given by $\bar{\varepsilon}=\sqrt{\epsilon_{a} \epsilon_{d}}$ and $\bar{D}=\sqrt{D_{a} D_{d}}$. Furthermore, with the above definitions of $\bar{\varepsilon}$ and $\bar{D}$, we can relate the parameter $l$ (defined here) to the parameter $\lambda$ (defined in Richardson et al. ${ }^{32}$ ) via $l=1 / \lambda^{2}$.

\section{Dimensionless equations}

The charge carrier conservation equations (2) and (3) non-dimensionalise to

$$
\frac{\partial \bar{p}}{\partial t}+\frac{\partial \bar{J}}{\partial \bar{x}}=0 \quad \text { in } \quad-1<\bar{x}<0
$$

and

$$
\frac{\partial \bar{n}}{\partial t}-\frac{\partial \bar{J}}{\partial \bar{x}}=0 \quad \text { in } \quad 0<\bar{x}<m .
$$

Since our primary objective here is to derive expressions for the current-voltage curves of a cell at steady state, it is enough to note that these equations and the continuity of current implied by the conditions (9) integrate to imply that $\bar{J}$ is constant throughout the device. It follows that the dimensionless steady state equations read

$$
\left.\begin{array}{c}
\kappa\left(\frac{\partial \bar{p}}{\partial \bar{x}}+\bar{p} \frac{\partial \bar{\phi}}{\partial \bar{x}}\right)=-\bar{J} \\
\hat{\varepsilon} \frac{\partial^{2} \bar{\phi}}{\partial \bar{x}^{2}}=-\bar{p}
\end{array}\right\} \text { in }-1<\bar{x}<0,
$$

with jump and boundary conditions

$$
\begin{gathered}
\left.\bar{\phi}\right|_{\bar{x}=0^{-}}=\left.\bar{\phi}\right|_{\bar{x}=0^{+}},\left.\quad \hat{\hat{\varepsilon}} \frac{\partial \bar{\phi}}{\partial \bar{x}}\right|_{\bar{x}=0^{-}}=\left.\frac{1}{\hat{\hat{\varepsilon}}} \frac{\partial \bar{\phi}}{\partial \bar{x}}\right|_{\bar{x}=0^{+}}, \\
\bar{J}=\delta\left(\frac{\left.\left.\bar{n}\right|_{\bar{x}=0^{+}} \bar{p}\right|_{\bar{x}=0^{-}}-\imath^{2}}{\theta+\left.U \bar{n}\right|_{\bar{x}=0^{+}}+\left.(1-U) \bar{p}\right|_{\bar{x}=0^{-}}}\right)-\hat{G}, \\
\left.\bar{p}\right|_{\bar{x}=-1}=\frac{l}{N_{-}} \exp \left(\frac{\Phi_{b i}}{2}\right),\left.\quad \bar{\phi}\right|_{\bar{x}=-1}=\frac{\Phi-\Phi_{b i}}{2}, \\
\left.\bar{n}\right|_{\bar{x}=m}=\imath N_{-} \exp \left(\frac{\Phi_{b i}}{2}\right),\left.\quad \bar{\phi}\right|_{\bar{x}=m}=-\frac{\Phi-\Phi_{b i}}{2} .
\end{gathered}
$$

Henceforth, we drop the over-bar notation.

\section{Parameter estimates for real devices}

Here, we look to estimate the parameters in the model based on a pentacene $/ \mathrm{C}_{60}$ bilayer device fabricated by Credgington et al. ${ }^{6}$ Hole mobility in single crystal pentacene has been measured by Knipp ${ }^{19}$ as $4 \times 10^{-4} \mathrm{~m}^{2} \mathrm{~V}^{-1} \mathrm{~s}^{-1}$ (corresponding to a value of $D_{d}=10^{-6} \mathrm{~m}^{2} \mathrm{~s}^{-1}$ ), and a figure for electron mobility in $\mathrm{C}_{60}$ is given by Koster et $a l^{20}$ as $2.5 \times 10^{-7} \mathrm{~m}^{2} \mathrm{~V}^{-1} \mathrm{~s}^{-1}$ (corresponding to a value of $D_{a}=5 \times 10^{-9} \mathrm{~m}^{2} \mathrm{~s}^{-1}$ ). Conductivity measurements, however, are strongly sample dependent. Permittivity in $\mathrm{C}_{60}$ and pentacene are both about $4 \varepsilon_{0}$, and the half thickness of the bilayer cell fabricated by Credgington et al. $^{6}$ is $L=4 \times 10^{-8} \mathrm{~m}$. Using these figures and assuming that the cell operates at approximately $T=300 \mathrm{~K}$, we obtain

$$
\Pi_{0} \approx 4 \times 10^{21} \mathrm{~m}^{-3} \quad \text { and } \quad G_{0} \approx 10^{3} \mathrm{Am}^{-2} .
$$

This value of $G_{0}$ compares with the short circuit current density, at one Sun, measured by Credgington et al. ${ }^{6}$ of 
approximately $60 \mathrm{~A} \mathrm{~m}^{-2}$ suggesting that, at least for this device, $\hat{G}$, at one Sun, is relatively small at about $6 \times 10^{-2}$. However, it is important to recognise that the values of the electron and hole mobilities in a real bilayer device may be considerably lower than those measured in samples carefully prepared for the purpose of demonstrating the advantages of a particular material (such as those fabricated by Knipp ${ }^{19}$ ).

Given the relatively low current densities that can be obtained in organic bilayer solar cells, as a result of the inefficiency of exciton collection, they are best viewed as an experimental system that can be used to investigate the electrical behaviour of particular combinations of acceptor and donor materials. It is therefore pertinent to ask what happens at high illumination levels in which the current densities are more in line with those that would be observed in more efficient bulk-heterojunction devices.

Typical values of the permittivities and mobilities of acceptor and donor materials suggest that both $\hat{\varepsilon}$ and $\kappa$ are $O(1)$. We shall also take the value of $m, N_{-}, U$, and $\theta$ to be of $O(1)$. The parameter $l$ measures the ratio of the thermally generated electron (and hole) densities in the dark to those under illumination and we might reasonably expect it to be small; however, since there is little data on thermally generated electron (and hole) densities, and since the limit $l \rightarrow 0$ is a non-singular limit, we take it to be an $O(1)$ quantity for the purpose of the ensuing analysis. The parameter $\delta$ measures the ease with which recombination takes place at the acceptor-donor interface. If $\delta \ll 1$, recombination is hard, and in moderate forward bias, the device behaves as a diode (i.e., the current varies exponentially with voltage). However, if $\delta \gg 1$, recombination is easy, and in moderate forward bias, the device's behaviour is more akin to an Ohmic resistor (i.e., the current-voltage curve is linear and the dominant resistance is due to the motion of the charge carriers through the semiconductor-this is commonly called a large series resistance). Thus, if the device is to work as an efficient solar cell $\delta$ should be small in order to stop recombination of solar generated charges and the attendant energetic losses associated with this. Indeed, this is what the data presented in Credgington et al. and Koster et al. suggest. ${ }^{6,20}$ This motivates us to consider a distinguished asymptotic limit in which $\delta \ll 1$. Furthermore, we examine two different operating regimes and, in each, derive an asymptotically valid expression for the current, $\bar{J}$, in terms of the applied voltage, $\Phi$.

\section{Outline of the ensuing analysis}

In Secs. III-VI, we shall solve the steady state dimensionless equations (17)-(22) in the limit $\delta \rightarrow 0$ with $m, l$, $\hat{\varepsilon}, \kappa, \theta, U$, and $N_{-}$all $O(1)$. We consider two different asymptotic limits, as the size of $\bar{J}$ and $\hat{G}$ vary relative to $\delta$, with the aim of deriving a simple asymptotic expression for the current, $J$, in terms of the applied cell voltage, $\Phi$. In the interests of clarity, we begin by considering a symmetric device for which the solution to Eqs. (17)-(22) is considerably simplified. Later, in Sec. VI, we discuss how these techniques can be generalised to obtain the solution of the steady state equations (17)-(22) for non-symmetric devices.

\section{The symmetric bilayer}

Here, we consider a device for which $\hat{\varepsilon}=1, m=1$, $N_{-}=1, \kappa=1$ so that the solutions to Eqs. (17)-(22) have the symmetry $p(-x)=n(x)$ and $\phi(x)=-\phi(-x)$. This symmetry allows us to collapse the problem onto one half of the device. Here, we choose to consider the acceptor region in $0<x<1$ where the problem may be stated as

$$
\frac{\partial n}{\partial x}-n \frac{\partial \phi}{\partial x}=J \quad \text { and } \quad \frac{\partial^{2} \phi}{\partial x^{2}}=n,
$$

for $0<x<1$ subject to boundary conditions

$$
\begin{gathered}
\left.\phi\right|_{x=0}=0 \quad \text { and } \quad J=\left.\delta\left(\frac{n^{2}-\imath^{2}}{\theta+n}\right)\right|_{x=0}-\hat{G} \\
\left.n\right|_{x=1}=\imath \exp \left(\frac{\Phi_{b i}}{2}\right) \quad \text { and }\left.\quad \phi\right|_{x=1}=-\frac{\Phi-\Phi_{b i}}{2}
\end{gathered}
$$

In the limit $\delta \rightarrow 0$, there are two distinguished limits of the parameters $J$ and $\hat{G}$ (relative to $\delta$ ) that must be considered in order to generate the device's entire current-voltage curve, see Figure 3. Before examining these limits, we eliminate $n$ from the system by using Eq. (24b) to restate the problem in the form

$$
\begin{gathered}
\phi_{x x x}-\phi_{x x} \phi_{x}=J \quad \text { in } \quad 0<x<1 \\
\left.\phi\right|_{x=0}=0 \quad \text { and } \quad J=\left.\delta\left(\frac{\phi_{x x}^{2}-\iota^{2}}{\theta+\phi_{x x}}\right)\right|_{x=0}-\hat{G} \\
\left.\phi_{x x}\right|_{x=1}=\imath \exp \left(\frac{\Phi_{b i}}{2}\right) \quad \text { and }\left.\quad \phi\right|_{x=1}=-\frac{\Phi-\Phi_{b i}}{2} .
\end{gathered}
$$

Before proceeding with the analysis, we consider the nature of the solutions to Eq. (27).

\section{PHASE PLANE ANALYSIS OF THE SYMMETRIC BILAYER}

Here, we discuss the solutions to Eq. (27) in terms of its phase plane. This gives considerable insight into the asymptotic structure of the solution to Eqs. (27)-(29) in the small $\delta$ limit. We begin by performing the canonical rescaling

$$
x=\left(\frac{2}{|J|}\right)^{1 / 3} \hat{x}
$$

of Eq. (27) and its boundary conditions (28b) and (29a). Under this change of variables, the system transforms to

$$
\begin{gathered}
\phi_{\hat{x} \hat{x} \hat{x}}-\phi_{\hat{x} \hat{x}} \phi_{\hat{x}}=\frac{2 J}{|J|}, \\
\phi_{\left.\hat{x} \hat{x}\right|_{\hat{x}=0}=}\left(\frac{2}{|J|}\right)^{2 / 3} \\
\times\left(\frac{J+\hat{G}}{2 \delta}+\sqrt{\left(\frac{J+\hat{G}}{2 \delta}\right)^{2}+\left(\frac{\theta(J+\hat{G})}{\delta}+r^{2}\right)}\right),
\end{gathered}
$$




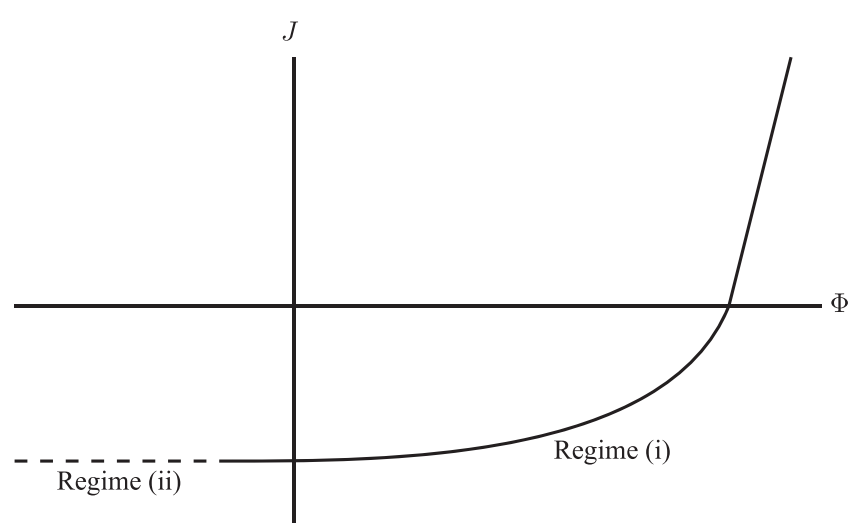

FIG. 3. A sketch of a typical current-voltage curve showing the ranges of validity of the two different asymptotic regimes.

$$
\left.\phi_{\hat{x} \hat{x}}\right|_{\hat{x}=(|J| / 2)^{2 / 3}}=\imath\left(\frac{2}{|J|}\right)^{2 / 3} \exp \left(\frac{\Phi_{b i}}{2}\right) .
$$

This is a second order autonomous boundary value problem for $\phi \hat{x}$. The general solution to Eq. (31) is , $^{2,32}$

$$
\phi=-2 \log \left|v_{1} \operatorname{Ai}\left(\hat{x}-\hat{x}_{0}\right)+v_{2} \operatorname{Bi}\left(\hat{x}-\hat{x}_{0}\right)\right|,
$$

where $\hat{x}_{0}, v_{1}$, and $v_{2}$ are arbitrary constants. However, the imposition of the boundary conditions on Eq. (34) results in a complicated set of non-trivial coupled transcendental equations. Rather than directly tackling these, it is more helpful to plot the phase portraits of Eq. (31). These are displayed in Figure 4 for the two possible cases $J>0$ (i.e., $J /|J|=1$ ) and $J<0$ (i.e., $J /|J|=-1$ ). In both panels, a dashed curve indicates the nullcline on which $\phi_{\hat{x} \hat{x} \hat{x}}=0$ and the solid curve the separatrix; the latter is a limiting solution given by $\phi$ $=-2 \log |\operatorname{Ai}(\hat{x})|$ that separates solutions for which either $n$ remains positive for all values of $\hat{x}$ from those for which $n$ passes through zero. Dotted curves are used to depict typical solutions lying on either side of the separatrix.

We note that Eq. (32) implies that there are no physically realistic solutions for $J \leq-\hat{G}-\delta \imath^{2} / \theta$ since such

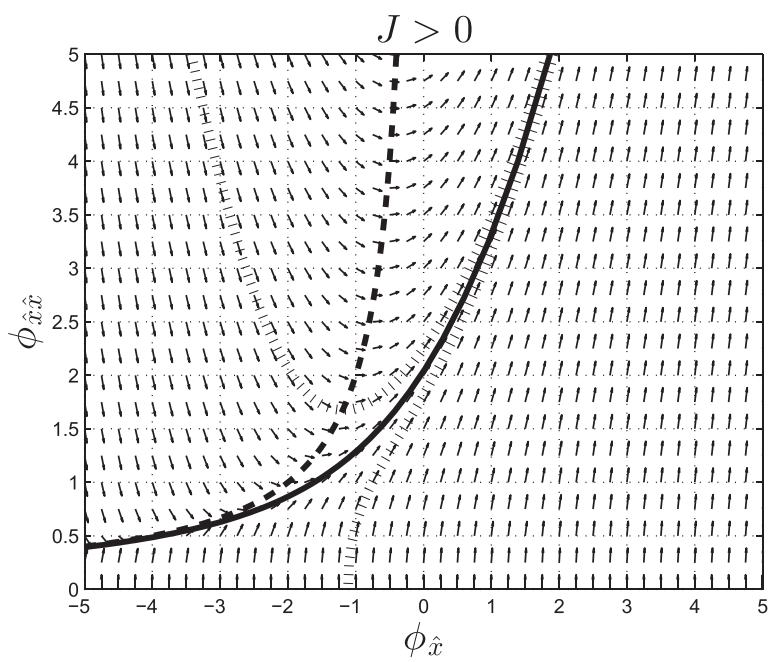

values correspond to a negative value of $\left.\phi_{\hat{x} \hat{x}}\right|_{\hat{x}=0}$, corresponding to a non-physical negative value of the electron density $n$ there. If however $J>-\hat{G}-\delta l^{2} / \theta$, then the boundary values of $\phi_{\hat{x} \hat{x}}$ are both positive (i.e., at $\hat{x}=0$ and $\left.\hat{x}=(|J| / 2)^{2 / 3}\right)$. In such scenarios, the nature of the phase planes plotted in 4 indicate that there are an infinite number of solution trajectories linking any two positive boundary values of $\phi_{\hat{x} \hat{x}}$, and along these trajectories, $\phi_{\hat{x} \hat{x}}$ is strictly positive (so that the solutions are physical). Furthermore, by choosing the appropriate trajectory, it is possible to obtain one along which $\hat{x}$ increases by exactly $(|J| / 2)^{2 / 3}$ so that, by specifying $\hat{x}=0$ at the start of this trajectory, the solution given by this trajectory satisfies the boundary conditions (32) and (33). Having found such a solution, it is trivial to enforce the extra condition $\left.\phi\right|_{\hat{x}=0}=0$ corresponding to Eq. (28a).

In the asymptotic analysis that follows, we consider the limit where $\delta$ is extremely small. It follows, except where the device is held in hard reverse bias, that $(J+\hat{G}) / \delta \gg 1$ and the boundary condition (32) can be approximated by

$$
\phi_{\hat{x} \hat{x}} \sim\left(\frac{2}{|J|}\right)^{2 / 3} \frac{J+\hat{G}}{\delta} \quad \text { on } \quad \hat{x}=0
$$

corresponding to a point on the phase plane far up the vertical axis. Various scenarios need then to be considered depending on whether $\imath \exp \left(\Phi_{b i} / 2\right)$ is comparably large and whether $J$ is positive or negative. All of these scenarios are susceptible to asymptotic analysis and will considered in Secs. IV-VI.

\section{ASYMPTOTIC ANALYSIS OF THE SYMMETRIC BILAYER AS $\delta \rightarrow 0$ (IDEALITY FACTOR 2)}

In Richardson et al., ${ }^{32}$ the problem of the symmetric cell was analysed in the limit $\delta \rightarrow 0$ with zero generation, i.e., $\hat{G}=0$. One of the main results was the derivation of the dark current-voltage curve. In addition, it was noted that the analysis can be trivially extended to describe small rates of generation (i.e., with $\hat{G}=O(\delta)$ ). In this section, we extend this analysis, in a non-trivial manner, to $O(1)$ generation rates and

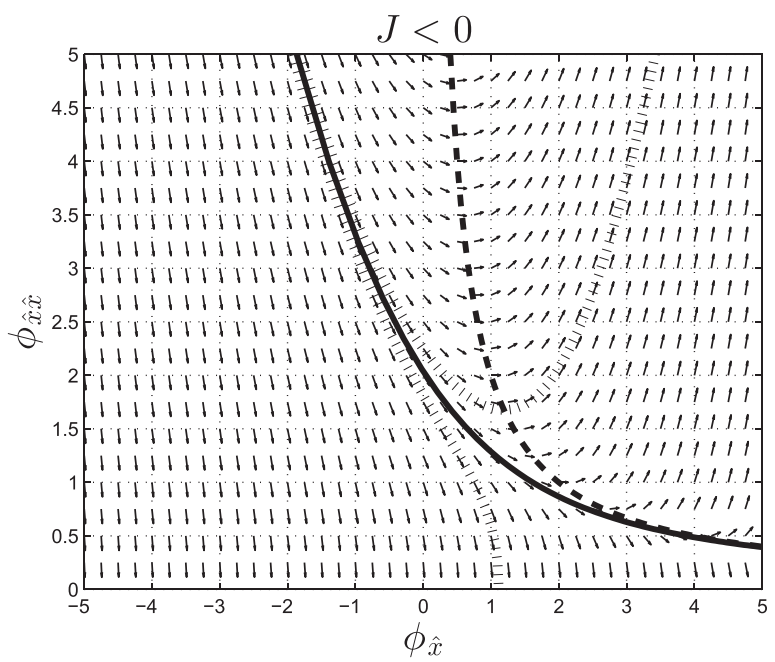

FIG. 4. The left and right panels show the phase plane for positive and negative $J$, respectively. Arrows indicate the direction of increasing $\hat{x}$. In both cases, the nullclines are indicated by a dashed curve, and the separatrix solution by a solid curve. Example phase paths (one on either side of the separatrix) are shown by dotted curves. 
above. This enables us to obtain current-voltage curves that are needed in order to describe the operation of a bilayer solar cell under strong illumination roughly consistent with one Sun and above. It will transpire that there are two distinguished limits that need to be considered in order to describe the entire current-voltage curve. The first of these (regime (ii) in Figure 3) describes the cell operating very close to reverse saturation and is therefore of little practical interest; however, for completeness, we provide a description in Sec. IV B. The second limit (regime (i) in Figure 3) describes the operation of the cell everywhere else, including the power generation regime, and will be treated in detail in the remainder of this section.

\section{A. Asymptotic regime (i)}

Here, we examine the behaviour of a symmetric device operating in a range of biases away from the reverse saturation current density. Formally, we consider $\hat{G}=O(1)$ and $J+\hat{G}=O(1)$. The approach adopted here will be to solve the ordinary differential equation (ODE) (27) for a given current, $J$, subject to the boundary conditions (28) and (29a) and then use the condition (29b) to determine the applied potential, $\Phi$, as a function of $J$. In this regime, analytic progress can be made due the presence of a boundary layer near the acceptor-donor interface in which the behaviour is dominated by the resistance of the interface. We begin by studying the structure within this layer.

\section{The inner region: Near the interface}

We investigate the boundary layer about $x=0$ by rescaling $x$ as follows:

$$
x=\frac{\delta^{1 / 2}}{(J+\hat{G})^{1 / 2}} \eta
$$

On substitution into Eqs. (27) and (28), this leads to the following problem for the potential in the inner region, $\phi^{i}$ :

$$
\begin{gathered}
\phi_{\eta \eta \eta}^{\mathrm{i}}-\phi_{\eta \eta}^{\mathrm{i}} \phi_{\eta}^{\mathrm{i}}=\frac{J \delta^{3 / 2}}{(J+\hat{G})^{3 / 2}}, \\
\left.\frac{\phi_{\eta \eta}^{\mathrm{i}}{ }^{2}-\delta^{2} \iota^{2} /(J+\hat{G})^{2}}{\phi_{\eta \eta}^{\mathrm{i}}+\delta \theta /(J+\hat{G})}\right|_{\eta=0}=1 \text { and }\left.\phi^{\mathrm{i}}\right|_{\eta=0}=0 .
\end{gathered}
$$

Expanding $\phi^{\mathrm{i}}$ as follows:

$$
\phi^{\mathrm{i}}=\phi_{0}^{\mathrm{i}}+O\left(\delta^{3 / 2} J(J+\hat{G})^{-3 / 2}\right),
$$

and substituting into Eqs. (37) and (38), we obtain, at leading order, a balance between drift and diffusive effects leading to the following problem for the leading order inner potential:

$$
\begin{gathered}
\phi_{0 \eta \eta \eta}^{\mathrm{i}}-\phi_{0 \eta \eta}^{\mathrm{i}} \phi_{0 \eta}^{\mathrm{i}}=0, \\
\left.\phi_{0}^{\mathrm{i}}\right|_{\eta=0}=0 \quad \text { and }\left.\quad \phi_{0 \eta \eta}^{\mathrm{i}}\right|_{\eta=0}=1,
\end{gathered}
$$

with solution

$$
\phi_{0}^{\mathrm{i}}=-2 \log \left(1+\frac{\eta}{2^{1 / 2}}\right)
$$

Matching the solution in the inner region (42) to the outer region leads to the following matching condition on the outer potential $\phi^{\circ}$ at leading and first order:

$$
\phi^{\mathrm{o}} \sim-\log \left(\frac{1}{\delta} \frac{J+\hat{G}}{2}\right)-2 \log x \quad \text { as } \quad x \rightarrow 0 .
$$

\section{The outer region}

Next, we consider the solution in the outer region where $x=O(1)$. Here, in order to match to the inner region via Eq. (43), the expansion proceeds as follows:

$$
\phi^{\mathrm{o}}=-\log \left(\frac{1}{\delta} \frac{J+\hat{G}}{2}\right)+\phi_{1}^{\mathrm{o}}+O\left(\delta^{1 / 2}(J+\hat{G})^{-1 / 2}\right) .
$$

On substitution of this expansion into Eqs. (27), (29), and (43), we obtain the following problem for $\phi_{1}^{o}$ the outer potential at first order:

$$
\phi_{1 x x x}^{\mathrm{o}}-\phi_{1 x x}^{\mathrm{o}} \phi_{1 x}^{\mathrm{o}}=J
$$

subject to

$$
\begin{gathered}
\phi_{1}^{\mathrm{o}} \sim-2 \log x \quad \text { as } \quad x \rightarrow 0, \\
\phi_{1 x x}^{\mathrm{o}}=\imath \exp \left(\frac{\Phi_{b i}}{2}\right) \quad \text { on } \quad x=1 .
\end{gathered}
$$

Notably, Eq. (45) is the full ODE. Furthermore, we can now see a distinction between the cases of $J>0$ and $J<0$, in which the form of the solution in the outer region changes as $J$ changes sign. Hence, we split the analysis into two parts (which follow) — the first for $J>0$ and the second for $J<0$.

a. The solution for $J>0$. In what follows, we solve the problems (45)-(47) for $\phi_{1}^{\mathrm{o}}$ with $J>0$. Imposing the matching condition (46) on the solution to Eq. (45) and noting that $\mathrm{Ai}(\cdot) \mathrm{Bi}^{\prime}(\cdot)-\mathrm{Bi}(\cdot) \mathrm{Ai}^{\prime}(\cdot)=1 / \pi$ gives the following expression for $\phi_{1}^{\mathrm{o}}$ :

$$
\begin{aligned}
\phi_{1}^{\mathrm{o}}= & -2 \log \mid \pi\left(\frac{2}{J}\right)^{1 / 3}\left(\operatorname{Bi}(w) \operatorname{Ai}\left(w-\left(\frac{J}{2}\right)^{1 / 3} x\right)\right. \\
& \left.-\operatorname{Ai}(w) \operatorname{Bi}\left(w-\left(\frac{J}{2}\right)^{1 / 3} x\right)\right) \mid .
\end{aligned}
$$

Here, $w$ is an as yet undetermined constant, $\operatorname{Ai}(\cdot)$ and $\operatorname{Bi}(\cdot)$ are Airy functions (defined in the usual way), and a prime denotes differentiation. Imposing the boundary condition (47) leads to the following transcendental equation that must be solved for $w$ :

$$
\begin{aligned}
& \left(\frac{\operatorname{Ai}(w) \operatorname{Bi}^{\prime}\left(w-\left(\frac{J}{2}\right)^{1 / 3}\right)-\operatorname{Bi}(w) \mathrm{Ai}^{\prime}\left(w-\left(\frac{J}{2}\right)^{1 / 3}\right)}{\operatorname{Bi}(w) \operatorname{Ai}\left(w-\left(\frac{J}{2}\right)^{1 / 3}\right)-\operatorname{Ai}(w) \operatorname{Bi}\left(w-\left(\frac{J}{2}\right)^{1 / 3}\right)}\right)^{2} \\
& =w-\left(\frac{J}{2}\right)^{1 / 3}+\frac{l}{2} \exp \left(\frac{\Phi_{b i}}{2}\right)\left(\frac{J}{2}\right)^{-2 / 3} .
\end{aligned}
$$


Although this transcendental equation must be solved numerically, we note that it is independent of $\hat{G}$. This drastically reduces the amount of computation needed to generate current-voltage curves for varying amounts of illumination since solutions for $w$ can be used for any number of different values of $\hat{G}$. Finally, we can read off the potential, $\Phi$, in terms of the current, $J$, by using Eq. (29b) to show that

$$
\Phi=2 \log \left(\frac{1}{\delta} \frac{J+\hat{G}}{2}\right)+\Phi_{b i}+P_{1}^{+}\left(J, \Phi_{b i}, \imath\right)
$$

where $P_{1}^{+}$is given by

$$
\begin{aligned}
P_{1}^{+}= & 4 \log \mid \pi\left(\frac{2}{J}\right)^{1 / 3}\left(\operatorname{Bi}(w) \operatorname{Ai}\left(w-\left(\frac{J}{2}\right)^{1 / 3}\right)\right. \\
& \left.-\operatorname{Ai}(w) \operatorname{Bi}\left(w-\left(\frac{J}{2}\right)^{1 / 3}\right)\right) \mid,
\end{aligned}
$$

and $w$ satisfies Eq. (49).

$b$. The solution for $J<0$. Here, we solve the problems (45)-(47) for $\phi_{1}^{\mathrm{o}}$ with $J<0$ and write $J=-|J|$. The structure of the analysis is broadly similar to the case $J>0$. The solution to Eq. (45), having imposing the matching condition (46), is

$$
\begin{aligned}
\phi_{1}^{\mathrm{o}}= & -2 \log \mid \pi\left(\frac{2}{|J|}\right)^{1 / 3}\left(\operatorname{Ai}(v) \operatorname{Bi}\left(v+\left(\frac{|J|}{2}\right)^{1 / 3} x\right)\right. \\
& \left.-\operatorname{Bi}(v) \operatorname{Ai}\left(v+\left(\frac{|J|}{2}\right)^{1 / 3} x\right)\right) .
\end{aligned}
$$

Here, the constant $v$ is determined by the condition (47) and thus satisfies the transcendental equation

$$
\begin{aligned}
& \left(\frac{\operatorname{Bi}(v) \mathrm{Ai}^{\prime}\left(v+\left(\frac{|J|}{2}\right)^{1 / 3}\right)-\operatorname{Ai}(v) \mathrm{Bi}^{\prime}\left(v+\left(\frac{|J|}{2}\right)^{1 / 3}\right)}{\operatorname{Bi}(v) \mathrm{Ai}\left(v+\left(\frac{|J|}{2}\right)^{1 / 3}\right)-\operatorname{Ai}(v) \mathrm{Bi}\left(v+\left(\frac{|J|}{2}\right)^{1 / 3}\right)}\right)^{2} \\
& =v+\left(\frac{|J|}{2}\right)^{1 / 3}+\frac{l}{2} \exp \left(\frac{\Phi_{b i}}{2}\right)\left(\frac{|J|}{2}\right)^{-2 / 3}
\end{aligned}
$$

Once $v$ has been determined the boundary condition (29b) can be used to find an expression for the potential, $\Phi$, in terms of the current, $J$,

$$
\Phi=2 \log \left(\frac{1}{\delta} \frac{\hat{G}-|J|}{2}\right)+\Phi_{b i}+P_{1}^{-}\left(|J|, \Phi_{b i}, l\right),
$$

where $P_{1}^{-}$is given by

$$
\begin{aligned}
P_{1}^{-}= & 4 \log \mid \pi\left(\frac{2}{|J|}\right)^{1 / 3}\left(\operatorname{Ai}(v) \operatorname{Bi}\left(v+\left(\frac{|J|}{2}\right)^{1 / 3}\right)\right. \\
& \left.-\operatorname{Bi}(v) \operatorname{Ai}\left(v+\left(\frac{|J|}{2}\right)^{1 / 3}\right)\right) \mid
\end{aligned}
$$

Here, we have considered a distinguished limit in which the solution to the problem has: (i) a narrow boundary layer about $x=0$ in which the current term in the potential equation (27) is unimportant. The behaviour in this layer gives rise to a logarithmic singularity in the far-field solution; (ii) an outer region in which the full potential equation and the full set of boundary conditions on the current collector (29) are satisfied together with a matching condition as $x \rightarrow 0$ (this matching condition is determined by the solution in the boundary layer). Provided that changes to the size of the parameters considered here leave this matching condition unaffected the results that we have obtained by analysing the outer problem are still applicable. In other words, the distinguished limit considered in Sec. IV A has a wide range of applicability. In particular, it can be used to obtain the current-voltage curve in the large current limit (see Sec. IV A 4) and the large built-in voltage limit (see Sec. IV A 6).

\section{The open-circuit voltage}

We can use the current voltage relations (50) and (54) to deduce an asymptotic expression for the dimensionless open-circuit applied voltage, $\Phi_{o c}$. In Appendix A, we show that when $J=0$,

$$
P_{1}^{+/-}\left(0, \Phi_{b i}, l\right)=2 \log \left(\frac{2}{l}\right)-\Phi_{b i},
$$

so that

$$
\Phi_{o c}=2 \log \left(\frac{\hat{G}}{\delta l}\right)
$$

Thus, the open-circuit voltage, $\Phi_{o c}$, varies logarithmically with the photo-current density $\hat{G}$ and is independent of the built-in voltage $\Phi_{b i}$ which is in agreement with previous studies. $^{4,37}$

\section{The large current limit $J \gg 1$}

We note that the distinguished limit treated in Sec. IV A is also valid for large positive currents. We can thus derive an approximate current-voltage relation in this limit simply by taking the expression (50) and determining the behaviour of $P_{1}^{+}\left(J, \Phi_{b i}\right)$ for $J \gg 1$ by finding the large $J$ asymptotic solution to Eq. (49) and substituting this into Eq. (51).

We begin by noting that the solution for $w$, obtained by solving Eq. (49) in the large $J$ limit, also becomes large and hypothesize that $w \gg(J / 2)^{1 / 3} \gg 1$. Since the large $z$ asymptotic behaviours of the Airy functions are given by

$$
\begin{aligned}
\operatorname{Ai}(z) \sim & \frac{\exp \left(-\frac{2}{3} z^{3 / 2}\right)}{2 \sqrt{\pi}}\left(z^{-1 / 4}-\frac{5}{48} z^{-7 / 4}\right), \\
\operatorname{Bi}(z) \sim & \frac{\exp \left(\frac{2}{3} z^{3 / 2}\right)}{\sqrt{\pi}}\left(z^{-1 / 4}+\frac{5}{48} z^{-7 / 4}\right),
\end{aligned}
$$

as $z \rightarrow \infty$, Eq. (49) can be approximated to exponential accuracy by 


$$
\left(\frac{\operatorname{Ai}^{\prime}\left(w-\left(\frac{J}{2}\right)^{1 / 3}\right)}{\operatorname{Ai}\left(w-\left(\frac{J}{2}\right)^{1 / 3}\right)}\right)^{2} \sim\left(w-\left(\frac{J}{2}\right)^{1 / 3}\right)+\frac{l e^{\Phi_{b i} / 2}}{2}\left(\frac{J}{2}\right)^{-2 / 3}
$$

Substituting for $\operatorname{Ai}\left(w-\left(\frac{J}{2}\right)^{1 / 3}\right)$ and its derivative from Eq. (58), we obtain the following asymptotic expression for $w$ :

$$
w \sim \frac{1}{l^{2}}\left(\frac{J}{2}\right)^{4 / 3} e^{-\Phi_{b i}} \quad \text { as } J \rightarrow \infty .
$$

Inserting this result into Eq. (51) yields an asymptotic expression for $P_{1}^{+}$,

$$
P_{1}^{+} \sim \frac{2 J}{l} \exp \left(-\frac{\Phi_{b i}}{2}\right) \quad \text { as } J \rightarrow \infty .
$$

\section{A uniformly valid asymptotic current voltage relation}

It is noteworthy that the terms $P_{1}^{+}$and $P_{1}^{-}$are relatively unimportant in the current voltage relationships (50) and (54), unless they are comparable in size to $\log (1 / \delta)$. Furthermore, we assert that this is never the case for $P_{1}^{-}$and occurs for $P_{1}^{+}$only when $J \geq O\left(l e^{\Phi_{b i} / 2} \log (1 / \delta)\right)$, where it is accurately described by the asymptotic relation (62). Nevertheless, when attempting to write down a uniformly valid asymptotic expansion for the current voltage relation, it is sensible to write down one for which the open circuit voltage $\Phi_{o c}$ is correct (albeit that this is a small correction to larger terms). The requisite uniformly valid description, which has the correct behaviours as $\Phi \rightarrow \pm \infty$ and which passes through $(\Phi, J)=\left(\Phi_{o c}, J\right)$, is

$$
\Phi=2 \log \left(\frac{\hat{G}+J}{\delta l}\right)+\frac{2 J}{\imath} \exp \left(-\frac{\Phi_{b i}}{2}\right) .
$$

This has the form of a current voltage relation obtained from a Shockley equivalent circuit with infinite shunt resistance (see Eq. (1) and Figure 2).

\section{The large built-in voltage limit $\Phi_{b i} \gg 1$}

The built-in potential $V_{b i}$ may, in practice, be considerably larger the thermal voltage $k T / q$ so that the dimensionless parameter $\Phi_{b i} \gg 1$. In this scenario, it is possible to make further analytical progress towards finding an approximate expression for the device's current-voltage curve. In a cell with a large built-in voltage, there is a boundary layer near the contact which arises due to the large value of $n$ there. However, there is no need to consider this layer explicitly in order to derive the solution and the current-voltage curve because, as in the large current case, the distinguished limit considered in Sec. IV A is uniformly valid for large built-in voltages. In order to find the asymptotic expressions for the terms $P_{1}^{+}\left(J, \Phi_{b i}\right)$ and $P_{1}^{-}\left(J, \Phi_{b i}\right)$ that appear in the current- voltage relations (50) and (54), respectively, it is necessary to find asymptotic solutions for $w$ and $v$, from Eqs. (49) and (53), respectively. We note that there are terms that are exponentially large in $\Phi_{b i}$ on the right-hand side of both of these equations, so that in order for there to be a balance with the terms on the left-hand side of these equations, we require that the denominators to be both close to zero; that is, $w$ and $v$ satisfy the asymptotic relations

$$
\begin{gathered}
\operatorname{Bi}(w) \operatorname{Ai}\left(w-\frac{J^{1 / 3}}{2^{1 / 3}}\right) \sim \operatorname{Ai}(w) \operatorname{Bi}\left(w-\frac{J^{1 / 3}}{2^{1 / 3}}\right) \\
\operatorname{Bi}(v) \operatorname{Ai}\left(v+\frac{|J|^{1 / 3}}{2^{1 / 3}}\right) \sim \operatorname{Ai}(v) \operatorname{Bi}\left(v+\frac{|J|^{1 / 3}}{2^{1 / 3}}\right),
\end{gathered}
$$

respectively. Solution to these relations yields values of $w$ and $v$ that can be substituted into Eqs. (51) and (55), respectively, to obtain expressions for $P_{1}^{+}$and $P_{1}^{-}$and hence to determine the current-voltage relations from Eqs. (50) and (54). Notably, the large $J$ limit of Eq. (64) is provided by the zeros of $\operatorname{Ai}\left(w-(J / 2)^{1 / 3}\right)$ such that $w \sim(J / 2)^{1 / 3}+z_{\text {ai }}$ where $z_{\text {ai }}$ is a zero of $\operatorname{Ai}(z)$. It is not until $J>O\left(\exp \left(3 \Phi_{b i} / 4\right)\right)$ that the large $J$ asymptotics become appropriate.

\section{B. Asymptotic regime (ii)}

Here, we consider the operation of the symmetric bilayer device close to the reverse saturation current density. Formally, we investigate the regime $J+\hat{G} \sim O(\delta)$ in which there is no boundary layer about the acceptor-donor interface at $x=0$. It follows that the behaviour of the device can only be described by solution to the full problem. However, since by definition $J \sim-\hat{G}$ and the current voltage curve is almost flat, the solution to this problem only yields the small correction to the current, obtained by varying the voltage and, hence, is of little interest. In fact, by examining Figure 5, one can observe that the asymptotic expressions (50) and (54) are uniformly valid in the interesting power generating regimes. In the interest of completeness, we state the problem describing the device behaviour in this particular limit, but do not solve it. The problem may be stated most simply by stating it in terms of the (small) perturbation to the current $\delta \hat{J}$ defined by

$$
J=-\hat{G}+\delta \hat{J}
$$

The leading order problem for the electric potential is thus

$$
\phi_{x x x}-\phi_{x x} \phi_{x} \sim-\hat{G}
$$

with

$$
\phi=0 \quad \text { on } \quad x=0
$$

and

$$
\phi_{x x}=\imath \exp \left(\frac{\Phi_{b i}}{2}\right) \text { and } \quad \phi=-\frac{\Phi-\Phi_{b i}}{2}
$$

on $x=1$. The solution to this third order problem for $\phi$ yields, on substitution into the recombination condition at the interface, an expression for the correction to the current 


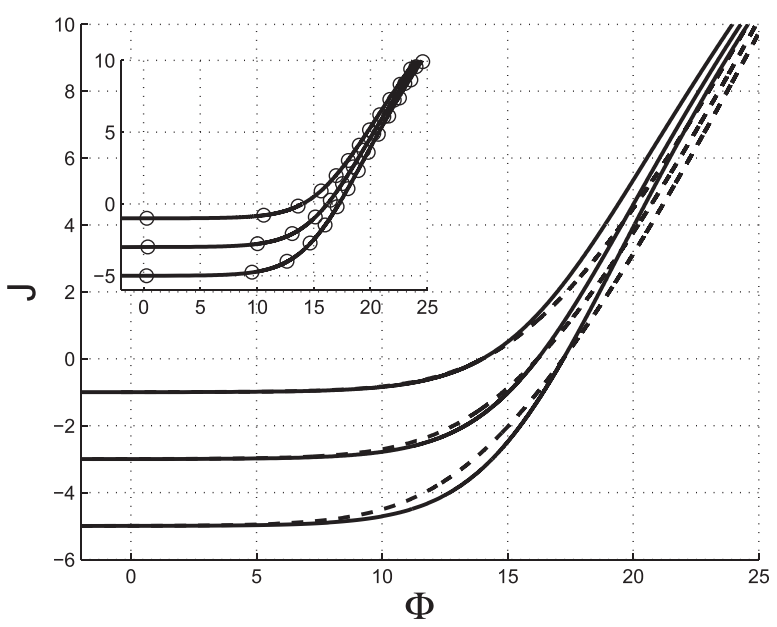

FIG. 5. Some typical current-voltage curve with $\delta=\exp (-7), \Phi_{b i}=1.1$, $\imath=1 \theta=1$, and $G=1,3$, and 5 . The circular markers, solid curves, and dashed curves indicate a full numerical solution of Eqs. (27)-(29), the asymptotic expressions (50) and (54), and the uniformly valid asymptotic approximation (63), respectively. Here, $\Phi$ is measured is units of thermal voltages $(\sim 0.026 \mathrm{~V})$. One unit of the $J$ corresponds to a current density given by the expression for $G_{0}$, see Eq. (13).

$$
\hat{J}=\left(\frac{\phi_{x x}^{2}-\imath^{2}}{\theta+\phi_{x x}}\right) \quad \text { on } \quad x=0 .
$$

We note that there is an analytic general solution to Eq. (67) and that application of the boundary conditions (68) and (69) provide sufficient conditions to determine the three constants in the general solution. One of these constants may be determined straightforwardly; however, the other two are determined from the solution to two coupled transcendental equations. In practice, it is probably not easier to solve these than to solve the original boundary value problem. However, in the limit $\hat{G} \gg 1$, it is possible to make further analytic progress. Here, the boundary-value problems (67)-(69) is tractable via matched asymptotic expansions in the two subregimes: (i) $\hat{G} \gg 1, J+\hat{G}=O\left(\delta \hat{G}^{2 / 3}\right)$ and (ii) $\hat{G} \gg$ $1, J+\hat{G}=O(\delta)$ that are required to complete the asymptotic description of large $\hat{G}$ regime.

\section{Comparison of asymptotic results to numerical results}

Here, we compare the asymptotics results derived above to numerical solutions of the problems (27)-(29). We obtain these numerical solutions by using the Chebfun (Ref. 9) (an open source library of algorithms which uses Chebyshev polynomials and automatic differentiation to solve ODE problems). Chebfun was selected because of its aptitude for accurately resolving stiff problems with solutions that vary rapidly within narrow boundary layers.

Figure 5 shows a comparison between current-voltage curves calculated using: (i) a full numerical solution of Eqs. (27)-(29) (circular markers); (ii) the asymptotic results (50) and (54) (solid curves); and (iii) the uniformly valid approximation (63) (dashed curves). The inset in Figure 5 shows extremely good agreement between the full numerical simulation and the asymptotic results (50) and (54). Further numerical experiments were carried out for a range of different parameters values, and extremely good agreement was always observed between asymptotic and numerical solutions. A comparison of the asymptotic results (50) and (54) to the uniformly valid asymptotic result (63) is shown in the main body of Figure 5. Despite this comparison being less favourable, the two families of curves are still asymptotic to one another in the limit that $\delta \rightarrow 0$. Importantly, the reverse saturation current density predicted by both sets of curves is consistent. Furthermore, the gradients of the curves for hard forward biases and the open-circuit voltages are in agreement. Figure 6 has also been included to show the model's prediction on the power generation of the device.

In Figure 7, typical charge carrier density and electric potential profiles are shown. These profiles were computed via a full numerical solution of Eqs. (27)-(29), and for the purpose of demonstration, we chose to use the same parameter values as the results shown in Figure 5 with $G=3$. They demonstrate Chebfun's ability to resolve solutions in boundary layers, where $n$ changes its value very rapidly. These plots also portray some features that one would expect to see that the charge carrier density at the interface increases as the device is pushed further into forward bias.

Finally, Figure 8 shows a comparison between a full numerical solution of Eqs. (27)-(29) and the asymptotic approximation for large built-in voltages, i.e., via (64) and (65). A good agreement between the two is observed. Other numerical experiments were carried out for different values of parameters and a good agreement was always observed.

\section{SMALL $\delta$ ASYMPTOTICS: EXTENSION TO IDEALITY FACTORS $\mathcal{N} \neq 2$}

Here, we investigate how alterations to the recombination and generation condition (9) affect the current-voltage curve. As discussed in Sec. II, it is common practice ${ }^{18}$ to adapt Eq. (9) to a condition of the form

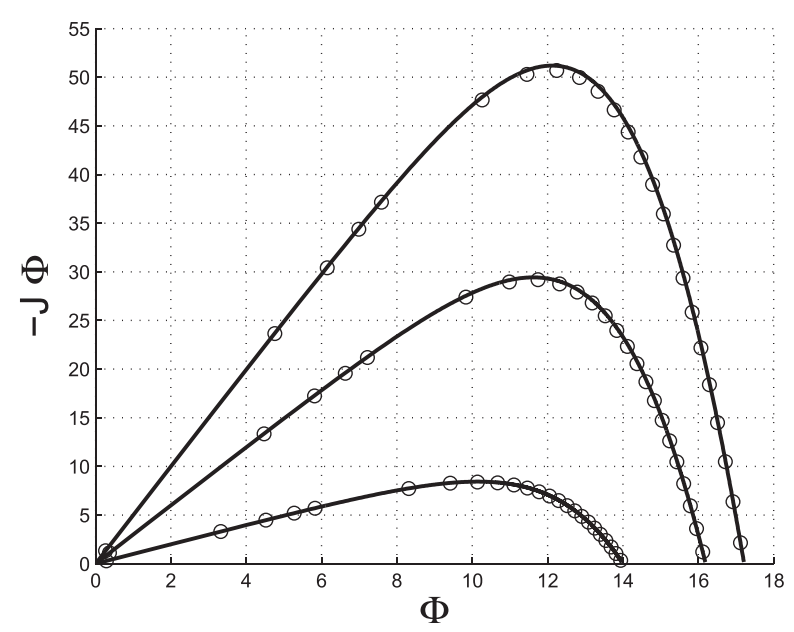

FIG. 6. Some power curves derived from the current-voltage curves shown in Figure 5. The solid curves are plots derived using the asymptotic expressions (50) and (54). The circular markers are results of a full numerical solution of Eqs. (27)-(29). Here, $\Phi$ is measured is units of thermal voltages $(\sim 0.026 \mathrm{~V})$. One unit of the $J$ corresponds to a current density given by the expression for $G_{0}$, see Eq. (13). 


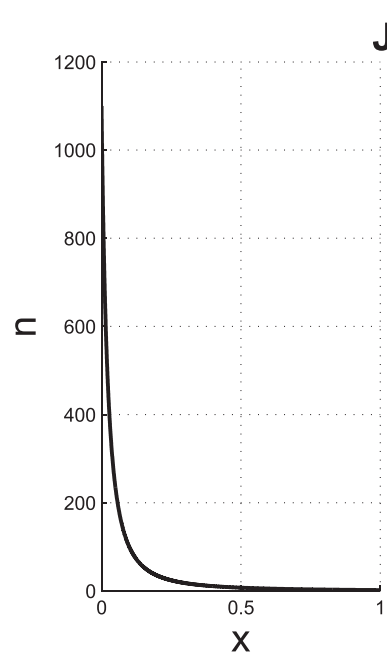

$J=-2$

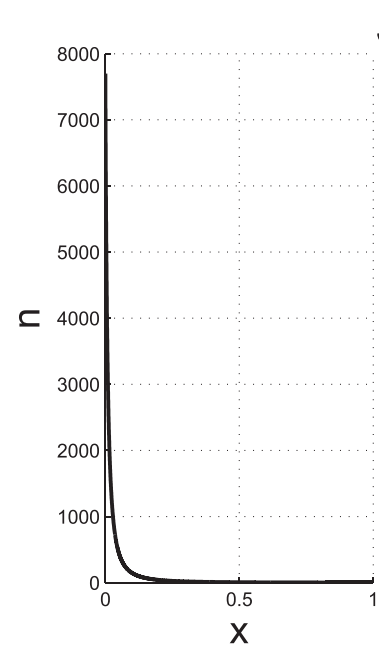

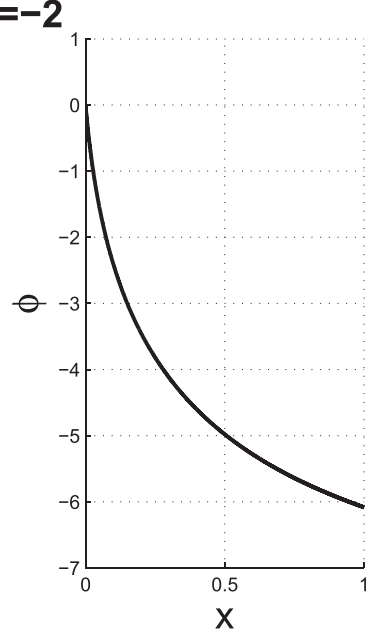

$\mathrm{J}=4$

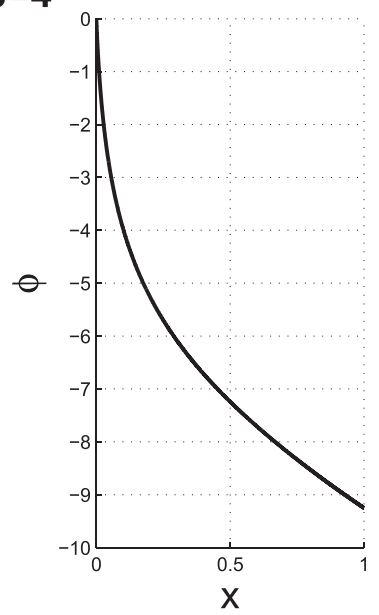

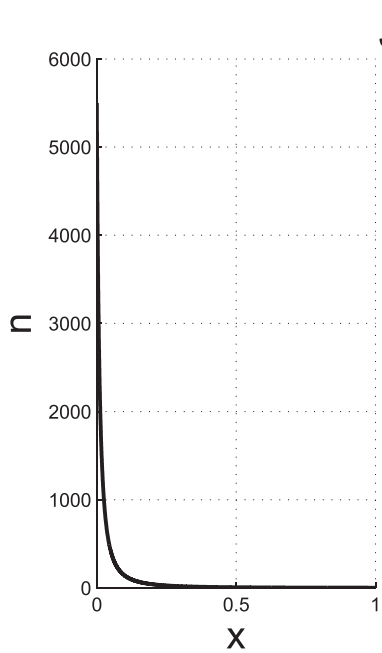

$\mathrm{J}=\mathbf{2}$
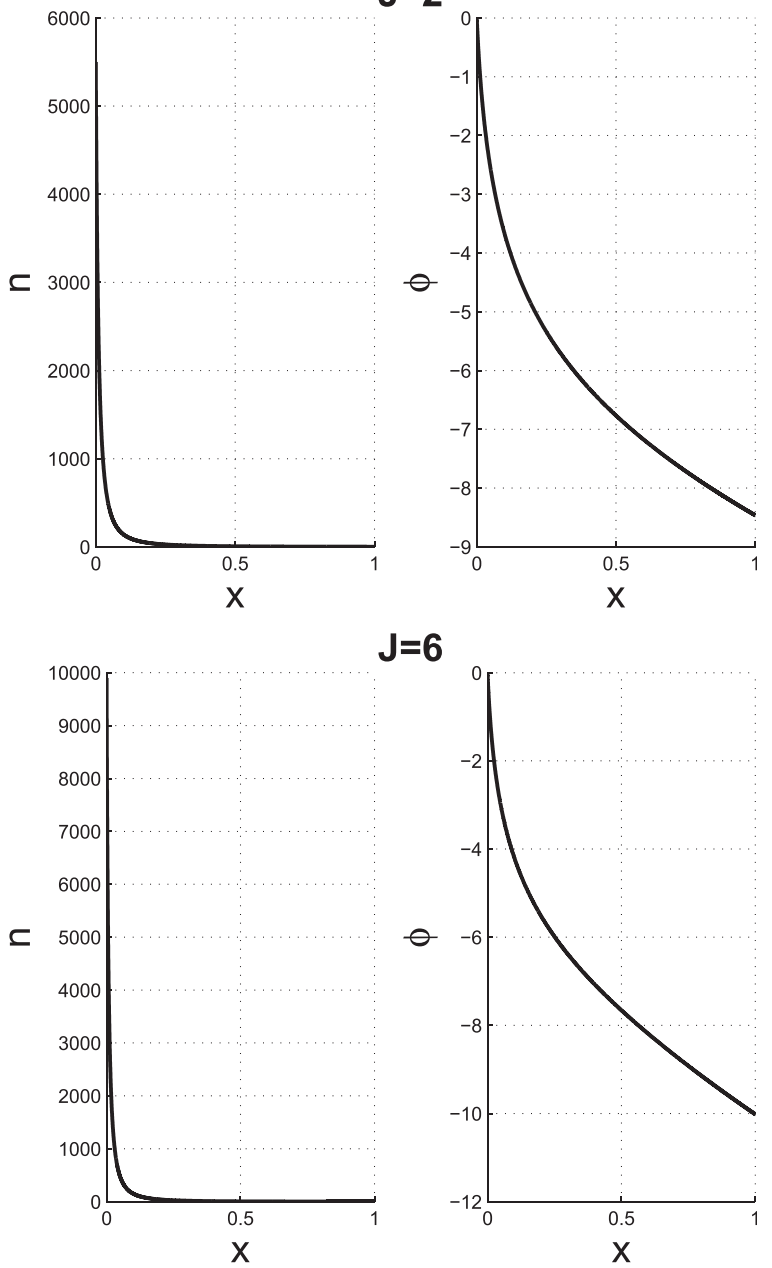

$\mathrm{J}=6$

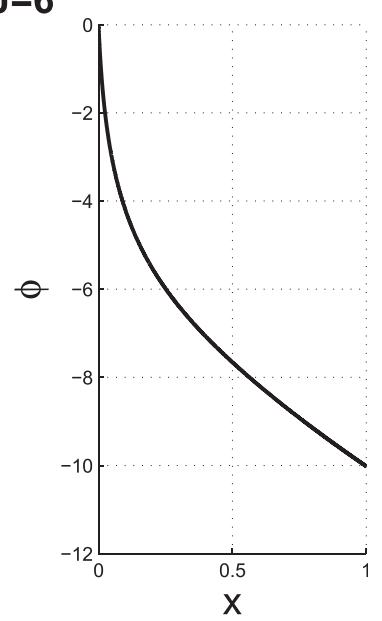

FIG. 7. Some example $n$ and $\phi$ profiles from the curve with $G=3$ shown in Figure 5. For the purposes of this demonstration, we chose the values of $J=-2,2$, 4 , and 6. Here, the potential, $\phi$, is measured as units of thermal voltages $(\sim 0.026 \mathrm{~V})$. One unit of the $n$ corresponds to an electron density given by the expression for $\Pi_{0}$, see Eq. (13).

$$
\begin{aligned}
\left.J\right|_{x=0^{-}}= & \left.J\right|_{x=0^{+}}=\left.\left.\mathcal{K} n^{\alpha-1}\right|_{x=0^{+}} p^{\beta-1}\right|_{x=0^{-}} \\
& \times\left(\left.\left.n\right|_{x=0^{+}} p\right|_{x=0^{-}}-N_{D}^{2} \exp \left(-E_{g} / k T\right)\right)-\hat{G}
\end{aligned}
$$

so that the case $\alpha=\beta=1$ corresponds to Langevin direct recombination. Here, $\mathcal{K}$ is a material parameter that is analogous to $K_{0}$ (although with different dimensions) in the case discussed in Sec. II. It has been shown in Kirchartz et al. ${ }^{18}$ that making this change to the recombination condition and selecting appropriate values for $\alpha$ and $\beta$ can lead to an improved fit to experimental data. In terms of the Shockley equivalent circuit formulation of the problem (see Figure 2 and Eq. (1)), this is equivalent to changing the ideality factor to $\mathcal{N}=2 /(\alpha+\beta)$. Thus, in the case $\alpha=\beta=1 / 2$, the results of this section are broadly similar to those of Sec. IV, in which we used a SRH recombination condition on the acceptordonor interface. Nondimensionalising Eq. (71) using Eq. (12) and assuming symmetry, as in Sec. IID (so that $\alpha=\beta$ ), lead to an adapted version of the original dimensionless symmetric bilayer model consisting of Eqs. (27), (28a), (29), and

$$
J=\hat{\delta} \phi_{x x}^{2 \alpha-2}\left(\phi_{x x}^{2}-\imath^{2}\right)-\hat{G} \quad \text { on } \quad x=0,
$$

where

$$
\hat{\delta}=\mathcal{K} \Pi_{0}^{\alpha+\beta} G_{0}^{-1} \ll 1
$$

plays an analogous role of the parameter $\delta$ in Sec. II. We now use the modified recombination condition (72) to derive a generalisation of the current-voltage relation obtained in Sec. IV for the symmetric bilayer with SRH recombination condition. Formally, we solve Eqs. (27), (28a), (29), and (72) in the limit $\hat{\delta} \rightarrow 0$. We begin by obtaining the asymptotic solution in an inner region close to $x=0$.

\section{A. The inner region: Near the interface}

Rescaling distance in Eqs. (27), (28a), and (72) by

$$
x=\frac{\hat{\delta}^{1 /(4 \alpha)}}{(J+\hat{G})^{1 /(4 \alpha)}} \chi
$$

leads to the following problem for the potential in the inner region:

$$
\begin{gathered}
\phi_{\chi \chi \chi}^{\mathrm{i}}-\phi_{\chi \chi}^{\mathrm{i}} \phi_{\chi}^{\mathrm{i}}=\frac{J \hat{\delta}^{3 /(4 \alpha)}}{(J+\hat{G})^{3 /(4 \alpha)}} \\
\left.\phi^{\mathrm{i}}\right|_{\eta=0}=0, \quad \phi_{\chi \chi}^{\mathrm{i} 2 \alpha}-\left.\frac{\hat{\delta}^{1 / \alpha} l^{2}}{(J+\hat{G})^{1 / \alpha}} \phi_{\chi \chi}^{\mathrm{i}{ }^{2 \alpha-2}}\right|_{\eta=0}=1 .
\end{gathered}
$$




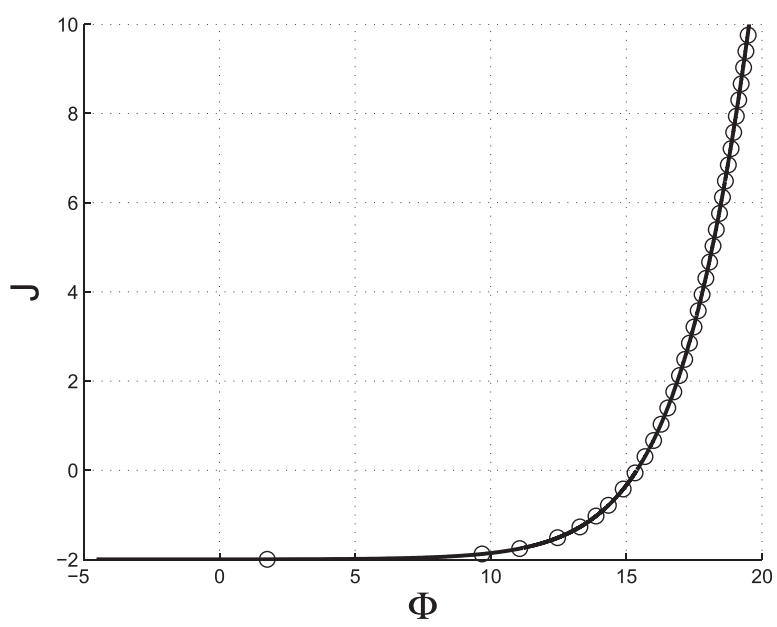

FIG. 8. A comparison of the large $\phi_{b i}$ approximations (64) and (65) (shown using the solid curve) with a full numerical solution of (27)-(29) (shown using circular markers). For the purposes of this demonstration, the following parameter values were selected: $\delta=\exp (-7), \Phi_{b i}=15, l=1$, and $\theta=1$. Here, $\Phi$ is measured as units of thermal voltages $(\sim 0.026 \mathrm{~V})$. One unit of the $J$ corresponds to a current density given by the expression for $G_{0}$, see Eq. (13).

Expanding $\phi^{\mathrm{i}}$ in powers of $\hat{\delta}$ as follows:

$$
\phi^{\mathrm{i}}=\phi_{0}^{\mathrm{i}}+O\left(\hat{\delta}^{3 /(4 \alpha)}, \hat{\delta}^{1 /\left(2 \alpha^{2}\right)}\right)
$$

yields the following problem for the leading order inner potential:

$$
\begin{gathered}
\phi_{0 \chi \chi \chi}^{\mathrm{i}}-\phi_{0 \chi \chi}^{\mathrm{i}} \phi_{0 \chi}^{\mathrm{i}}=0, \\
\phi_{0 \chi \chi}^{\mathrm{i}}=1, \quad \text { and } \phi_{0}^{\mathrm{i}}=0 \quad \text { on } \quad \chi=0 .
\end{gathered}
$$

This system has solution

$$
\phi_{0}^{\mathrm{i}}=-2 \log \left(1+\frac{\chi}{\sqrt{2}}\right) .
$$

Rewriting this solution in terms of the outer variable $x$ and expanding in powers of $\hat{\delta}$ yields a matching condition on the outer solution

$$
\phi_{0}^{\mathrm{i}} \sim-\frac{1}{2 \alpha} \log \left(\frac{1}{\hat{\delta}} \frac{J+\hat{G}}{2^{2 \alpha}}\right)-2 \log x \quad \text { as } \quad x \rightarrow 0 .
$$

\section{B. The outer region}

Motivated by the matching condition (81), we expand the potential in the outer region as follows:

$$
\phi^{\mathrm{o}}=-\frac{1}{2 \alpha} \log \left(\frac{1}{\hat{\delta}} \frac{J+\hat{G}}{2^{2 \alpha}}\right)+\phi_{1}^{\mathrm{o}}+\cdots .
$$

The problem to be satisfied by $\phi_{1}^{o}$ is

$$
\begin{gathered}
\phi_{1 x x x}^{\mathrm{o}}-\phi_{1 x x}^{\mathrm{o}} \phi_{1 x}^{\mathrm{o}}=J, \\
\phi_{1}^{\mathrm{o}} \sim-2 \log x \text { as } x \rightarrow 0,
\end{gathered}
$$

$$
\phi_{1 x x}^{\mathrm{o}}=\imath \exp \left(\frac{\Phi_{b i}}{2}\right) \quad \text { on } \quad x=1 .
$$

This is identical to the first order outer problem considered in Sec. IV A; namely, Eqs. (45)-(47). It follows that $\phi_{1}^{\mathrm{o}}$ is given by Eqs. (48) and (49) in the case $J>0$, and by Eqs. (52) and (53) in the case $J<0$. In turn, the current-voltage relationship is

$$
\Phi=\frac{1}{\alpha} \log \left(\frac{1}{\hat{\delta}} \frac{J+\hat{G}}{2^{2 \alpha}}\right)+\Phi_{b i}+P_{1}^{+}\left(J, \Phi_{b i}\right)
$$

for $J>0$, and

$$
\Phi=\frac{1}{\alpha} \log \left(\frac{1}{\hat{\delta}} \frac{J+\hat{G}}{2^{2 \alpha}}\right)+\Phi_{b i}+P_{1}^{-}\left(|J|, \Phi_{b i}\right)
$$

for $J<0$ where $P_{1}^{+}$and $P_{1}^{-}$are defined by Eqs. (50) and (54), respectively. An expression for the open-circuit voltage $\Phi_{o c}$ can be derived by setting $J=0$ in the above and is

$$
\Phi_{o c}=\frac{1}{\alpha} \log \left(\frac{\hat{G}}{\hat{\delta} l^{2 \alpha}}\right) .
$$

The large $J$ asymptotics of $P_{1}^{+}$is given by Eq. (62) and it follows that (since the $P_{1}^{+}$and $P_{1}^{-}$are small in comparison to the logarithmic terms in Eqs. (86) and (87), respectively, unless $J \gg 1$ ) a uniformly valid expansion for $\Phi$ (that passes through $\left.(\Phi, J)=\left(\Phi_{o c}, 0\right)\right)$ is

$$
\Phi=\frac{1}{\alpha} \log \left(\frac{J+\hat{G}}{\hat{\delta} l^{2 \alpha}}\right)+\frac{2 J}{l} \exp \left(-\frac{\Phi_{b i}}{2}\right) .
$$

\section{NON-SYMMETRIC DEVICES}

In this section, we discuss how the results from Secs. III-V can be generalised to a bilayer device without any inherent symmetry. We concentrate the analysis on the most practically relevant operating regime; that is, regime (i) which occurs when $\hat{G}=O(1)$ and $J+\hat{G} \gg O(\delta)$ (for the case of a symmetric device, this was studied in Sec. IV A). To derive an expression for the current-voltage of a bilayer cell without any inherent symmetry one must solve the problems (17)-(22).

The problems (17)-(22) can be solved analytically using matched asymptotics and one can show that the structure of the solution bears many similarities to the solution found in Sec. IV A, i.e., there is a boundary layer close to, and, either side of the acceptor-donor interface (where drift and diffusive effects are dominant), and in the outer regions (in the bulk of both the acceptor and donor), the full ODEs must be solved. Although the solution to Eqs. (17)-(22) can be written down, it depends on four complicated, coupled transcendental equations. Consequently, it is difficult to interpret meaningful features of the solution and so one could argue that a more insightful (and straight-forward) approach would be to solve the systems (17)-(22) entirely numerically. Of course finding a numerical solution presents its own 
challenges; however, they can be overcome by using an appropriate numerical algorithm (see Sec. IV C for details). This being said, it is relatively straight-forward to show that

$$
\begin{aligned}
& \Phi=2 \log \left(\frac{1}{\delta} \frac{J+\hat{G}}{q^{+}}\right)+\Phi_{b i}+Q_{1}^{+} \quad \text { for } J>0, \\
& \Phi=2 \log \left(\frac{1}{\delta} \frac{J+\hat{G}}{q^{-}}\right)+\Phi_{b i}+Q_{1}^{-} \quad \text { for } J<0 .
\end{aligned}
$$

Here $q^{+}, q^{-}$are constants, and $Q_{1}^{+}$and $Q_{1}^{-}$are functions of $J$ and $\Phi_{b i}$ (note that all four of these quantities depend on the material properties of the cell). From the results (90) and (91) alone, one can interpret some insightful properties of a general bilayer cell. Foremost, one can see that the dominant term, in both the non-symmetric and symmetric cell's current voltage curve, is of the form $\Phi=2 \log ((J+\hat{G}) / \delta)$. However, for devices with extreme asymmetries, it is possible that the terms $Q_{1}^{+/-}$become comparable in size to the leading term. Previous studies have shown that this can lead to $S$-shaped current-voltage curves ${ }^{26,39}$ — a fact that we have independently verified. Another situation that can lead to $S$-shaped currentvoltage curves is when the equilibrium charge carrier densities at the contacts are very small. In this case, if a positive current is to be passed through the device, holes (or electrons) must be extracted from the donor (or acceptor) contact, and since the equilibrium number of charge carriers at the contacts is small, this can act to limit current flow.

Figure 9 shows a comparison of the asymptotic results (90) and (91) (solid curves) with a full numerical solution to Eqs. (17)-(22) (circular markers). Discussion of the numerical scheme is given in Sec. IV C. The numerical solutions were fitted to Eqs. (90) and (91) by taking $q^{+}$and $q^{-}$to be unity, $Q_{1}^{-}$ to be zero (since its value does not significantly influence the shape of the current-voltage curve), and $Q_{1}^{+}$to be a linear

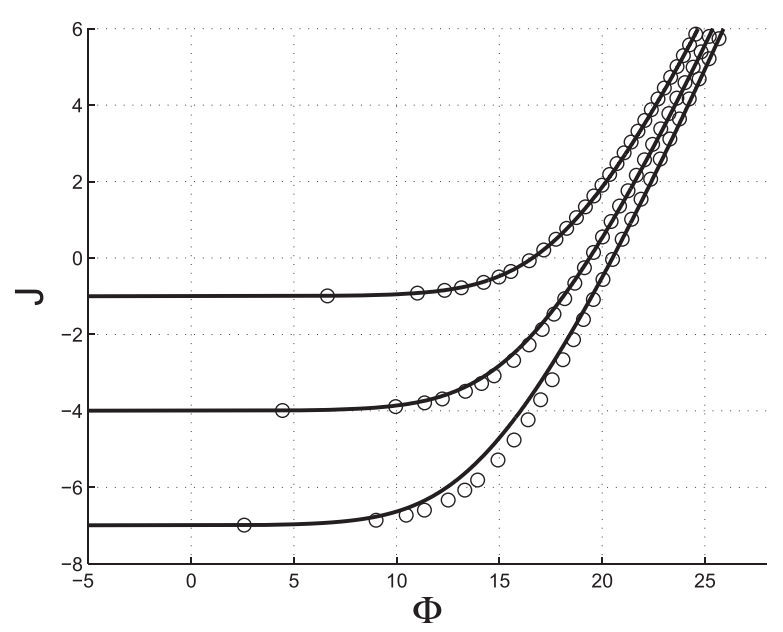

FIG. 9. A comparison of a numerical solution for a non-symmetric device, i.e., Eqs. (17)-(22) (shown using circular markers) and the asymptotic expressions (90) and (91) (shown using solid curves). For the purposes of this demonstration, we selected the following parameter values: $\delta=\exp (-8), \Phi_{b i}$ $=2, l=1, \theta=1, \epsilon=1, \kappa=1, m=1.8, N_{-}=1, U=1 / 3$, and $G=1,4$, and 7. Here, $\Phi$ is measured is units of thermal voltages $(\sim 0.026 \mathrm{~V})$. One unit of the $J$ corresponds to a current density given by the expression for $G_{0}$, see Eq. (13). function of $J$. This procedure is a generalisation of the arguments presented in Sec. IV A 5 to the non-symmetric device. We found that taking $Q_{1}^{+} \sim 0.695 \mathrm{~J}$ in the asymptotic theory gave a good fit to the numerical results as can be seen from Figure 9.

\section{CONCLUSIONS}

In this work, we considered a drift-diffusion model for a bilayer organic solar cell consisting of a layer of donor material abutting a layer of acceptor and sandwiched between two electrical contacts. In our treatment of the problem, we neglected the effects of minority carriers (i.e., electrons in the donor and holes in the acceptor) arguing, on the basis of the large jumps in ionization potential and electron affinity between electron and donor, that their effects are insignificant. In the instance of a perfectly symmetric device with SRH recombination (ideality factor $\mathcal{N}=2$ ), we used asymptotic methods to investigate the resulting model (in Sec. IV) and obtained a simple current-voltage relation. In Sec. V, we extended the analysis to symmetric devices with an alternative recombination mechanism that yields ideality factors other than 2 . In both cases, we were able to find a uniformly valid asymptotic expression for the current-voltage relation that could be expressed in the form

$$
\bar{J} \sim \hat{\delta} \imath^{2 / \mathcal{N}} \exp \left(\frac{\Phi-(2 / \imath) e^{-\Phi_{b i} / 2} \bar{J}}{\mathcal{N}}\right)-\hat{G}
$$

where $\mathcal{N}=2$ with $\mathrm{SRH}$ recombination (see Sec. IV A and Eq. (63)) and $\mathcal{N}=1 / \alpha$ where the generalised recombination law described in Sec. $\mathrm{V}$ is used (see Eq. (89)). Redimensionalising this result, via Eqs. (12)-(14) and (73), yields the following expression [in the case of SRH recombination we take $\left.\mathcal{K}=K_{0} /\left(u_{1}+u_{2}\right)\right]$ :

$$
\begin{aligned}
J \sim & -G+\mathcal{K} N_{D}^{2 / \mathcal{N}} \exp \left(-\frac{E_{g}}{\mathcal{N} k T}\right) \\
& \times \exp \left(\frac{V-\frac{2 k T L}{q^{2} N_{D} \sqrt{D_{a} D_{d}}} \exp \left(-\frac{q V_{b i}-E_{g}}{2 k T}\right) J}{\mathcal{N} k T / q}\right)
\end{aligned}
$$

that we can now compare to the formula for the SEC model (see Figure 2)

$$
\mathcal{I}=\frac{R_{p}}{R_{p}+R_{s}} \times\left(\mathcal{I}_{0}\left[\exp \left(\frac{\mathcal{V}-\mathcal{I} R_{s} A}{\mathcal{N} k T / q}\right)-1\right]-\mathcal{G}-\frac{\mathcal{V}}{R_{p} A}\right)
$$

in the limit that the shunt resistance $R_{p} \rightarrow \infty$. We can thus identify $\mathcal{N}$ as the ideality factor; $\mathcal{K} N_{D}^{2 / \mathcal{N}} \exp \left(-E_{g} / \mathcal{N} k T\right)$ as the reverse saturation current density $\mathcal{I}_{0}$; and $2 k T L \exp \left(-\left(q V_{b i}-E_{g}\right) /(2 k T)\right) /\left(q^{2} N_{D} \sqrt{D_{a} D_{d}}\right)$ as the series resistance multiplied by the area of the cell $A R_{s}$. Note that the reverse saturation current density term $\mathcal{I}_{0}$ does not appear explicitly in the asymptotic expression (93) since it is swamped by the photo-current density term $G$. In practice, one could incorporate a non-infinite shunt resistance into our model by including the effect of (i) minority carriers or allowing for (ii) the occurrence of pinhole defects in the 
bilayer. ${ }^{11,22}$ The former mechanism would lead to a nonlinear shunt resistance that depends upon the applied potential since the density of minority carriers is much higher in forward bias than in reverse bias (examples of organic photovoltaic models that include effects due to minority carriers are given in Refs. 1 and 2). Furthermore, the measured values of ionization potential and electron affinity differences between typical acceptor and donor materials are extremely large (as measured in units of $k T$ ) suggesting that this mechanism is not responsible for any observed shunt resistances. It is therefore more likely that significant shunt resistances result from pinhole defects in the bilayer, whereby one of the semiconductors directly contacts both electrodes leading to a local short circuit. A natural extension of this work would therefore be to include pinholes into the model geometry.

To demonstrate the comparison between our model and the SEC model, we have included Figure 10 which shows some current-voltage curves for devices of varying thicknesses. In line with the discussion above, this variation in thickness corresponds to varying the series resistance. Notably, Figure 10 shows that the open-circuit voltage is independent of the device's width. In addition, we can observe that an increase in the thickness (or equivalently the series resistance) reduces the fill factor of the device- a well known result. ${ }^{25}$

We obtained numerical solutions to the model by use of a numerical package Chebfun, ${ }^{9}$ based on Chebyshev polynomials. This approach allowed us to deal with what is often an extremely stiff problem that exhibits very rapid growth in narrow boundary layers. In the case of a symmetric cell, numerical solutions to the model compared very favourably with our asymptotic solutions (see Sec. IV C). In Sec. VI, this numerical approach was used to investigate asymmetric cells, which are appreciably more awkward to treat with asymptotic methods than symmetric cells (due to necessary but inherently cumbersome algebra). Sample current voltage curves were generated for several different parameter sets and fitted to a Shockley

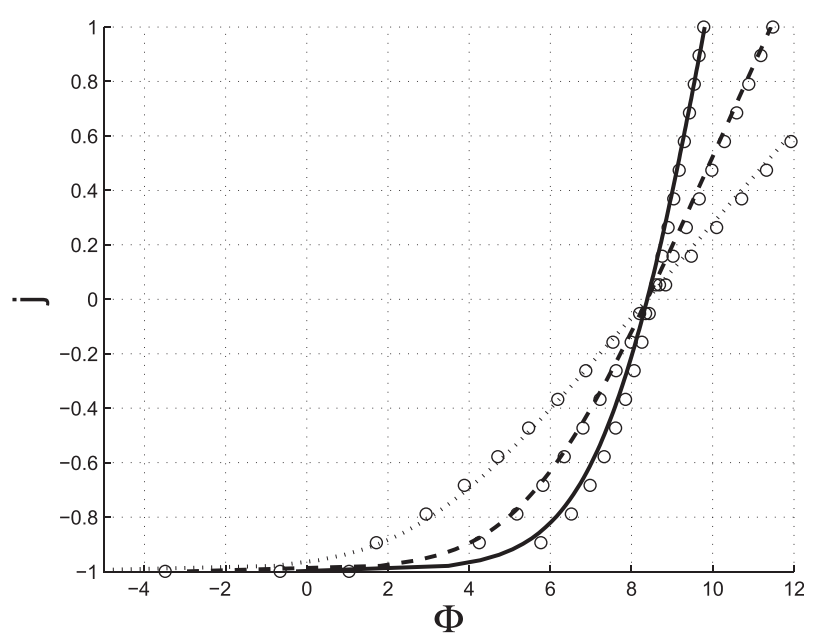

FIG. 10. Current-voltage curves for symmetric devices of three different half widths $L, 2 L$, and $3 L$ (indicated by solid, dashed, and dotted curves, respectively), of ideality factor $\mathcal{N}=1$ and with the same dimensional photoinduced current $G=\sqrt{\epsilon_{d} \epsilon_{a}} \sqrt{D_{d} D_{a}} k T / q L^{3}$. All other properties of the devices were also held constant. Here, the dimensionless current $j$ (measured in units of $\left.q L^{3} J /\left(\sqrt{\epsilon_{d} \epsilon_{a}} \sqrt{D_{d} D_{a}} k T\right)\right)$ is plotted versus the dimensionless voltage $\Phi$ (measured in units of thermal voltages, $\sim 0.026 \mathrm{~V}$ ). The circular markers were determined using the uniformly valid asymptotic expression (89). equivalent circuit model (with infinite shunt resistance) and, as in the symmetric case, the comparison was good.

\section{ACKNOWLEDGMENTS}

J.F. and G.R. would both like to thank the EPSRC, who funded this research through Grant No. EP/I01702X/1, and Colin Please and an anonymous referee for helpful comments. This publication is partially based on work supported by Award No. KUK-C1-013-04, made by King Abdullah University of Science and Technology (KAUST), via an OCCAM visiting research fellowship awarded to G.R.

\section{APPENDIX A: SOLUTION OF THE OPEN-CIRCUIT VOLTAGE PROBLEM}

Here we give some details on the solution of (44)-(47) with $J=0$, the open-circuit voltage problem. We have

$$
\phi^{\mathrm{o}}=-\log \left(\frac{1}{\delta} \frac{\hat{G}}{2}\right)+\phi_{1}^{\mathrm{o}}+O\left(\delta^{1 / 2} \hat{G}^{-1 / 2}\right) .
$$

On substitution of this expansion into Eqs. (27), (29), and (43), we obtain the following problem for $\phi_{1}^{\text {o }}$ the outer potential at first order:

$$
\phi_{1 x x x}^{\mathrm{o}}-\phi_{1 x x}^{\mathrm{o}} \phi_{1 x}^{\mathrm{o}}=0,
$$

subject to

$$
\begin{gathered}
\phi_{1}^{\mathrm{o}} \sim-2 \log x \quad \text { as } \quad x \rightarrow 0, \\
\phi_{1 x x}^{\mathrm{o}}=\imath \exp \left(\frac{\Phi_{b i}}{2}\right) \quad \text { on } \quad x=1 .
\end{gathered}
$$

We begin by noting that there are two solutions to Eq. (A2) that satisfy the matching condition (A3), namely,

$$
\begin{aligned}
& \phi_{1}^{\mathrm{o}}=-2 \log \left(\frac{\sin \left(\omega_{1} x\right)}{\omega_{1}}\right), \\
& \phi_{1}^{\mathrm{o}}=-2 \log \left(\frac{\sinh \left(\omega_{2} x\right)}{\omega_{2}}\right) .
\end{aligned}
$$

Imposing the boundary condition (A4), we find that the solution (A5) is the appropriate when $\imath \exp \left(\Phi_{b i} / 2\right)>2$, whereas, if $\imath \exp \left(\Phi_{b i} / 2\right)<2$, the solution (A6) is required. By virtue of the fact that the second derivatives of Eqs. (A5) and (A6) on $x=1$ are $2 \omega_{1}^{2} / \sin ^{2}\left(\omega_{1}\right)$ and $2 \omega_{2}^{2} / \sinh ^{2}\left(\omega_{2}\right)$, respectively, we can immediately write

$$
\left.\phi_{1}^{\mathrm{o}}\right|_{x=1}=-\log \left(\frac{2}{l}\right)+\frac{\Phi_{b i}}{2} .
$$

Finally, recalling the boundary condition (26b) and resubstitution of Eq. (A7) into Eq. (A1) quickly give the following expression for the open-circuit voltage:

$$
\Phi_{o c}=2 \log \left(\frac{\hat{G}}{\delta l}\right) .
$$


${ }^{1}$ G. A. Buxton and N. Clarke, "Computer simulation of polymer solar cells," Modell. Simul. Mater. Sci. Eng. 15, 13-26 (2007).

${ }^{2}$ D. Brinkman, K. Fellner, P. A. Markowich, and M. T. Wolfram, "A driftdiffusion-reaction model for excitonic photovoltaic bilayers: Asymptotic analysis and a 2-D HDG finite-element scheme," Math. Models Meth. Appl. Sci. 23, 839-872 (2013).

${ }^{3}$ J. A. Barker, C. M. Ramsdale, and N. C. Greenham, "Modelling the currentvoltage characteristics of bilayer polymer photovoltaic devices," Phys. Rev. B 67, 075205 (2003).

${ }^{4}$ D. Cheyns, J. Heremans, P. Deibel, C. Verlaak, S. Rand, and J. Genoe, "Analytical model for the open-circuit voltage and its associated resistance in organic planar heterojunction solar cells," Phys. Rev. B 77, 165332 (2008).

${ }^{5}$ J. D. Cole, "Limit process expansions and homogenization," SIAM J. Appl. Math. 55, 410-424 (1995).

${ }^{6}$ D. Credgington, Y. Kim, J. Labram, T. D. Anthopoulos, and J. R. Durrant, "Analysis of recombination losses in a Pentacene/ $\mathrm{C}_{60}$ organic bilayer," J. Phys. Chem. Lett. 2, 2759-2763 (2011).

${ }^{7}$ B. K. Crone, P. S. Davids, I. H. Campbell, and D. L. Smith, "Device model investigation of bilayer organic light emitting diodes," J. Appl. Phys. 87, 1974 (2000).

${ }^{8}$ P. S. Davids, I. H. Campbell, and D. L. Smith, "Device model for single carrier organic diodes," J. Appl. Phys. 82, 6319 (1997).

${ }^{9}$ L. N. Trefethen et al., Chebfun Version 4.2, The Chebfun Development Team, 2011, see http://www.maths.ox.ac.uk/chebfun/.

${ }^{10} \mathrm{C}$. de Falco, R. Sacco, and M. Verri, "Analytical and numerical study of photocurrent transients in organic polymer solar cells," Comput. Meth. Appl. Mech. Eng. 199, 1722-1732 (2010).

${ }^{11}$ D. Gebeyehu, C. J. Brabec, F. Padinger, T. Fromherz, J. C. Hummelen, D. Badt, H. Schindler, and N. S. Sariciftci, "The interplay of efficiency and morphology in photovoltaic devices based on interpenetrating networks of conjugated polymers with fullerenes," Synth. Met. 118, 1-9 (2001).

${ }^{12}$ K. A. Gregg and M. C. Hanna, "Comparing organic to inorganic photovoltaic cells: Theory, experiment, and simulation," J. Appl. Phys. 93, 3605-3614 (2003).

${ }^{13}$ C. Groves, J. C. Blakesley, and N. C. Greenham, "Effect of charge trapping on geminate recombination and polymer solar cell performance," Nano Lett. 10, 1063-1069 (2010).

${ }^{14}$ S. Günes, H. Neugebauer, and N. S. Sariciftci, "Conjugated polymerbased organic solar cells," Chem. Rev. 107, 1324 (2007).

${ }^{15}$ H. Hoppe and N. S. Sariciftci, "Organic solar cells: An overview," J. Mater. Res. 19, 1924-1945 (2004).

${ }^{16} \mathrm{P}$. E. de Jongh and D. Vanmaekelbergh, "Trap-limited transport in assemblies of nanometer-size $\mathrm{TiO}_{2}$ particles," Phys. Rev. Lett. 77, 3427-3430 (1996).

${ }^{17}$ B. Kippelen and D. Vanmaekelbergh, "Organic photovoltaics," Energy Environ. Sci. 2, 251-261 (2009).

${ }^{18}$ T. Kirchartz, B. E. Pieters, J. Kirkpatrick, U. Rau, and J. Nelson, "Recombination of tail states in polythiopene: Fullerene solar cells," Phys. Rev. B. 83, 115209 (2011).

${ }^{19} \mathrm{D}$. Knipp, "Pentacene thin film transistors on inorganic dielectrics: Morphology, structural properties, and electronic transport," J. Appl. Phys. 93, 347-355 (2003)

${ }^{20}$ L. J. A. Koster, E. C. P. Smits, V. D. Mihailetchi, and V. W. M. Blom, "Device model for the operation of polymer/fullerine bulk heterojunction solar cells," Phys. Rev. B. 72, 085205 (2005).
${ }^{21}$ J. Kirkpatrick, V. Marcon, K. Kremer, J. Nelson, and D. Andrienko, "Charge mobility in discotic mesophases: A multiscale quantum and classical study," Phys. Rev. Lett. 98, 227402 (2007).

${ }^{22}$ F. C. Krebs, "Fabrication and processing of polymer solar cells: A review of printing and coating techniques," Sol. Energy Mater Sol. Cells 93, 394-412 (2009)

${ }^{23}$ A. Manor, E. A. Tromholt, and F. C. Krebs, "Electrical and photo-induced degradation of $\mathrm{ZnO}$ layers in photovoltaics," Adv. Energy Mater. 1, 836-843 (2011)

${ }^{24}$ C. M. Martin, V. M. Burlakov, H. E. Assender, and D. A. R. Barkhouse, "A numerical model for explaining the role of the interface morphology in composite solar cells," J. Appl. Phys. 102, 104506 (2007).

${ }^{25}$ J. Nelson, The Physics of Solar Cells (Imperial College Press, 2003).

${ }^{26} \mathrm{~J}$. Nelson, J. Kirkpatrick, and P. Revirajan, "Factors limiting the efficiency of molecular photovoltaic devices," Phys. Rev. B 69, 035337 (2004).

${ }^{27}$ T. Offermans, S. C. J. Meskers, and R. A. J. Janssen, "Monte-Carlo simulations of geminate electron-hole pair dissociation in a molecular heterojunction: A two step dissociation mechanism," Chem. Phys. 308, 125-133 (2005).

${ }^{28}$ P. Peumans, S. Uchida, and S. R. Forrest, "Efficient bulk heterojunction photovoltaic cells using small-molecular-weight organic thin films," Nature 425, 158-162 (2003).

${ }^{29}$ W. J. Potscavage, S. Yoo, and B. Kippelen, "Origin of the open-circuit voltage in a multillayer heterojunction organic solar cell," Appl. Phys. Lett. 93, 193308 (2008).

${ }^{30}$ L. Pautmeier, R. Richert, and H. Bassler, "Poole-Frenkel behaviour of charge transport in organic solid with off-diagonal disorder studied by Monte-Carlo simulations," Synth. Met. 37, 271 (1990).

${ }^{31}$ G. Richardson, G. Denuault, and C. P. Please, "Multiscale modelling and analysis of lithium-ion battery charge and discharge," J. Eng. Math. 72, 41-72 (2012)

${ }^{32}$ G. Richardson, C. Please, J. Foster, and J. Kirkpatrick, "Asymptotic solution of a model for bilayer organic diodes and solar cells," SIAM J. Appl. Math. 72, 1792-1817 (2012).

${ }^{33}$ G. G. Malliaras and J. C. Scott, "The roles of injection and mobility in organic light emitting diodes,” J. Appl. Phys. 83, 5399-5403 (1998).

${ }^{34} \mathrm{~B}$. Stuart, "Heliatek achieves 10.7 percent OPV efficiency; to begin module production this year," PV magazine (2012).

${ }^{35}$ S. M. Sze and K. N. Kwok, Physics of Semiconductor Devices (WileyInterscience, New York, 2006).

${ }^{36} \mathrm{C}$. W. Tang, "Two-layer organic photovoltaic cell," Appl. Phys. Lett. 48, 183-185 (1986).

${ }^{37} \mathrm{~W}$. Tress, K. Leo, and M. Riede, "Influence of hole-transport layers and donor materials on open-circuit voltage and shape of I-V curves of organic solar cells," Adv. Funct. Mater. 21, 2140-2149 (2011).

${ }^{38} \mathrm{~W}$. Tress, K. Leo, and M. Riede, "Photoconductivity as a loss mechanism in organic solar cells," Phys. Status Solidi (RRL) 7, 401-405 (2013).

${ }^{39}$ W. Tress, A. Petrich, M. Hummert, M. Hein, K. Leo, and M. Riede, "Imbalanced mobilities causing S-shaped IV curves in planar heterojunction solar cells," Appl. Phys. Lett. 98, 063301 (2011).

${ }^{40}$ S. Verlaak, D. Beljonne, D. Cheyns, C. Rolin, M. Linares, F. Castet, J. Cornil, and P. Heremans, "Electronic structure and geminate pair energetics at organic-organic interfaces: The case of pentacene/ $\mathrm{C}_{60}$ heterojunctions," Adv. Funct. Mater. 19, 3809-3814 (2009). 